\title{
Targeting tumor-intrinsic hexosamine biosynthesis sensitizes pancreatic cancer to anti-PD1 therapy
}

\author{
Nikita S. Sharma, Vineet K. Gupta, Vanessa T. Garrido, Roey Hadad, Brittany C. Durden, Kousik Kesh, Bhuwan Giri, \\ Anthony Ferrantella, Vikas Dudeja, Ashok Saluja, and Sulagna Banerjee
}

Department of Surgery and Sylvester Comprehensive Cancer Center, University of Miami, Miami, Florida, USA.

\begin{abstract}
Pancreatic ductal adenocarcinoma (PDAC) is considered to be a highly immunosuppressive and heterogenous neoplasm. Despite improved knowledge regarding the genetic background of the tumor and better understanding of the tumor microenvironment, immune checkpoint inhibitor therapy (targeting CTLA4, PD1, PDL1) has not been very successful against PDAC. The robust desmoplastic stroma, along with an extensive extracellular matrix (ECM) that is rich in hyaluronan, plays an integral role in this immune evasion. Hexosamine biosynthesis pathway (HBP), a shunt pathway of glycolysis, is a metabolic node in cancer cells that can promote survival pathways on the one hand and influence the hyaluronan synthesis in the ECM on the other. The rate-limiting enzyme of the pathway, glutamine-fructose amidotransferase 1 (GFAT1), uses glutamine and fructose 6 -phosphate to eventually synthesize uridine diphosphate $\mathrm{N}$-acetylglucosamine (UDP-GIcNAc). In the current manuscript, we targeted this glutamine-utilizing enzyme by a small molecule glutamine analog (6-diazo-5-oxo-L-norleucine [DON]). Our results showed that DON decreased the self-renewal potential and metastatic ability of tumor cells. Further, treatment with DON decreased hyaluronan and collagen in the tumor microenvironment, leading to an extensive remodeling of the ECM and an increased infiltration of CD8 ${ }^{+}$T cells. Additionally, treatment with DON sensitized pancreatic tumors to anti-PD1 therapy, resulting in tumor regression and prolonged survival.
\end{abstract}

\section{Introduction}

Pancreatic cancer is a devastating disease with very poor outcomes. In spite of concerted efforts to develop effective therapeutic strategies, the 5-year survival rate of patients diagnosed with this disease is a mere $7 \%(1,2)$. High rates of tumor relapse, therapeutic resistance, and metastatic spread of the tumor contribute to these dismal statistics $(3,4)$. Compounding this, the pancreatic tumor is largely immune evasive. This makes these tumors resistant to immune checkpoint inhibitor therapies (with anti-PD1, anti-PDL1, and anti-CTLA4 therapy) that have shown remarkable benefit in other tumors $(5,6)$. Resistance to these immune therapies is predominantly due to the presence of an extensive fibroinflammatory and desmoplastic stroma with a rich extracellular matrix (ECM). Together, these components of the tumor provide an immunesuppressive microenvironment that prevents the infiltration of antitumor immune cells, such as $\mathrm{CD}^{+} \mathrm{T}$ cells $(7,8)$. Targeting the stromal component (such as activated stellate cells, cancerassociated fibroblasts (CAFs), or the hyaluronan-rich [HA-rich] ECM) has shown sensitization of pancreatic tumors to standard

\section{Related Commentary: p. 71}

Conflict of interest: University of Minnesota has a patent for Minnelide (WO/ 2010/129918), which has been licensed to Minneamrita Therapeutics LLC. AS is the cofounder and the chief scientific officer of this company. SB is a consultant with Minneamrita Therapeutics LLC, and this relationship is managed by the University of Miami. Copyright: () 2020, American Society for Clinical Investigation.

Submitted: January 17, 2019; Accepted: October 8, 2019; Published: December 3, 2019. Reference information: J Clin Invest. 2020;130(1):451-465.

https://doi.org/10.1172/JCI127515. chemotherapy over recent years (9-12). However, it is acknowledged that an antistromal therapy by itself is likely to result in a more aggressive tumor by relieving pressure on blood vessels and promoting metastasis (13). Thus, a treatment strategy that can deplete the stroma while eradicating the tumor cells in order to prevent metastatic spread is ideal for this devastating disease.

While the role of stellate cells in secretion of the robust ECM is well known (7), how the ECM may contribute to the immunesuppressive microenvironment within pancreatic tumor remains an enigma. Studies have shown that infiltration of $\mathrm{CD}^{+} \mathrm{T}$ cells is associated with better patient outcomes $(14,15)$. Similarly, depletion of $\mathrm{CD} 4^{+} \mathrm{T}$ cells in pancreatic cancer mouse models has been shown to result in decreased infiltration of several tumor-promoting myeloid cell populations, such as macrophages and immature myeloid cells or myeloid-derived suppressor cells (MDSCs), indicating that the immune cell behavior within the tumor microenvironment is a complex interaction between different cell types $(16,17)$. In patients with pancreatic cancer, an immune response is typically mediated by $\mathrm{CD}^{+} \mathrm{T}$ cell infiltration. However, a large number of these cells become trapped in the peritumoral stroma and do not reach the tumor to elicit an antitumor response (18). Migration of infiltrating $\mathrm{T}$ cells is typically guided by stromal chemokines and ECM proteins $(19,20)$. Additionally, T cells use the ECM fibers to migrate to tumor cells whether by ameboid-like contact guidance or using integrin-based adhesion $(20,21)$. Thus, remodeling of ECM is likely to have a profound effect on $\mathrm{T}$ cell infiltration and function in pancreatic cancer (22).

The ECM of pancreatic tumors is primarily composed of glycosaminoglycans such as HA. HA is a polymer of glucuronic acid 
(GlcUA) and $N$-acetylglucosamine (GlcNAc). Synthesis of HA is orchestrated by HA synthases (HAS1-3). These enzymes require uridine diphosphate-GlcNAc (UDP-GlcNAc) as one of the primary substrates for synthesis of HA $(23,24)$. In a cell, UDP-GlcNAc is synthesized via the hexosamine biosynthesis pathway (HBP), a shunt pathway of glycolysis that utilizes glutamine and glucose to make this nucleic acid sugar $(25,26)$. Since HBP is also a nutrient-sensing pathway, the synthesis of HA in these tumors is tightly coupled with the metabolic status of the cells. The rate-limiting enzyme of this pathway is glutamine-fructose amidotransferase $1 / 2$ (GFAT1/2), which is responsible for controlling the flux of metabolites through this pathway. The HBP is highly utilized in a number of cancers, and its inhibition by either targeting GFAT1/2 or by preventing utilization of glutamine (using a glutamine analog, 6-diazo-5-oxo-Lnorleucine [DON]) results in tumor regression (27-29).

UDP-GlcNAc is a major metabolite that is used in cellular glycosylation reactions. Decreased UDP-GlcNAc production can induce ER stress in the cells by inhibiting these reactions (30). Further, since UDP-GlcNAc is used by O-GlcNAc transferase (OGT) to glycosylate and regulate a large number of oncogenic proteins (such as Myc), inhibition of UDP-GlcNAc production will affect the survival of the cancer cells (31). Consistent with this, previous studies from our laboratory have shown that HBPfueled UDP-GlcNAc synthesis can be used by OGT to drive tumor growth and self-renewal $(32,33)$. Thus, HBP forms an integral metabolic node, inhibition of which will affect the tumor and its microenvironment alike. While the role of HBP in cancer cell survival is being studied by several groups, whether HBP can be targeted genetically or pharmacologically to remodel the ECM, and thus make the immune evasive pancreatic tumor susceptible to immune therapy, has not been studied before. Since the HBP is dependent on glucose uptake and glutamine equally, inhibition of glutamine utilization by using glutamine analogs such as azaserine and DON has been used over the years to target this pathway. Even though studies have shown that DON may have pleiotropic effects (34), it was used clinically as an antitumor agent (35). DON was successfully used in 5 phase 2 clinical trials (34), leading to disease stabilization. However, in spite of showing promise, DON was abandoned clinically as an antitumor agent. With the recent knowledge that tumor microenvironment is an integral component that drives progression of the tumors, there has been a renewed interest in reviving DON as an antitumor agent. Since recent evidence showed that a broad-spectrum antagonist of glutamine is more effective in inducing tumor regression than selective inhibition of a single glutamine-utilizing enzyme (36), DON is being reevaluated as a potential therapy in a number of cancers, specifically in combination with other chemotherapeutic agents $(37,38)$. Therapeutic strategies using DON against glutaminedependent tumors have also been proposed (38).

Owing to the central role of HBP in pancreatic cancer, which is known to be heavily dependent on glutamine metabolism (39), we evaluated DON in pancreatic cancer as both an antitumor and antistromal agent in the current study. Our results showed that DON acted as a potent antitumor agent and inhibited self-renewal and clonogenicity in pancreatic cancer cells. It also decreased metastatic potential of pancreatic cancer cells substantially. Further, when coimplanted with pancreatic cancer-associated fibroblasts in the pancreas of C57BL/6 mice, DON had a profound effect on the ECM and promoted infiltration of $\mathrm{CD} 8^{+}$cytotoxic T cells. Further, infiltration of cytotoxic $\mathrm{T}$ cells in pancreatic tumors following DON administration also sensitized them to anti-PD1 therapy. Since pancreatic tumors are notoriously immune evasive, this observation is extremely promising, as it indicates that metabolic inhibitors such as DON can be developed to overcome immune resistance and improve survival rates in this disease

\section{Results}

$H B P$ is overactivated in pancreatic cancer. Since chronic pancreatitis is a well-known risk factor for pancreatic ductal adenocarcinoma (PDAC) (40), we studied the expression of GFAT1/2 (alias GFPT1/2) as well as the other enzymes in this pathway in caerulein-induced chronic pancreatitis as well as in a KRAS ${ }^{\mathrm{G} 12 \mathrm{D}} \mathrm{P} 53^{\mathrm{R} 172 \mathrm{H}} \mathrm{Pdx}-1$-Cre (KPC) pancreatic cancer mouse model during tumor progression. Our results showed that these enzymes were overexpressed upon induction of pancreatitis (Figure 1A) as well as during pancreatic tumor progression (Figure 1B). In addition, expression of GFAT1 was also increased in the ductal cells of the pancreatic adenocarcinoma when observed in a tumor tissue microarray (Figure 1C). Further, an analysis of The Cancer Genome Atlas (TCGA) database showed that this pathway was overexpressed in $35.7 \%$ of the 176 pancreatic cancer patients in the database at both the RNA and protein levels (Figure 1D). To study whether GFAT1 was expressed both in the tumor and the stroma, we performed immunohistochemistry with anti- $\alpha$-SMA and anti-GFAT1 Ab. Our results showed that GFAT1 was predominantly expressed in the tumor cells. As shown in Figure 2, GFAT1 did not costain with $\alpha$-SMA in the mouse KPC tumors (Figure 2A) or in the human tumors (Figure 2B). Since GFAT1 is the rate-limiting step of this pathway, we focused our study on this particular enzyme.

GFAT1 contributed to aggressive biology of pancreatic cancer by regulating self-renewal and metastasis. A mark of an aggressive tumor is its ability to metastasize and its potential to relapse after treatment. These are dependent on the genes that regulate self-renewal. Our previous results (32) showed that OGT, an enzyme dependent on UDP-GlcNAc and thus HBP, was instrumental in regulating self-renewal in pancreatic cancer via its effect on SOX2. Our results showed that inhibition of GFAT1, the rate-limiting enzyme of $\mathrm{HBP}$, using siRNA resulted in inhibition of a number of self-renewal genes, such as SOX2, OCT4, and $K L F 4$, in the pancreatic cancer cell lines MIA-PaCa2 and S2VP10 (Figure 3A). Since GFAT1 activity is dependent on the availability of glutamine, we next blocked glutamine utilization with DON. Our studies showed that treatment of pancreatic cancer cell lines MIA-PaCa2 and S2VP10 with DON resulted in decreased expression of self-renewal genes, as seen with GFAT siRNA (Supplemental Figure 1A; supplemental material available online with this article; https://doi.org/10.1172/JCI127515DS1). To study whether the inhibition of HBP by blocking glutamine utilization with DON resulted in decreased clonogenicity (a surrogate assay for selfrenewal), we performed a colony-forming assay on the pancreatic cancer cell line S2VP10, which is aggressive and has high selfrenewal capability. Our results showed that treatment with DON resulted in decreased colony formation, showing that glutamine utilization by HBP was instrumental in decreasing self-renewal in 
A Hexosamine biosynthesis pathway

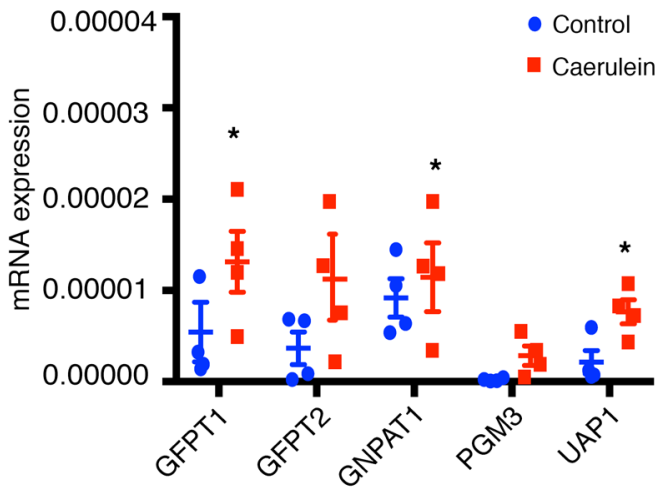

B

Hexosamine biosynthesis pathway

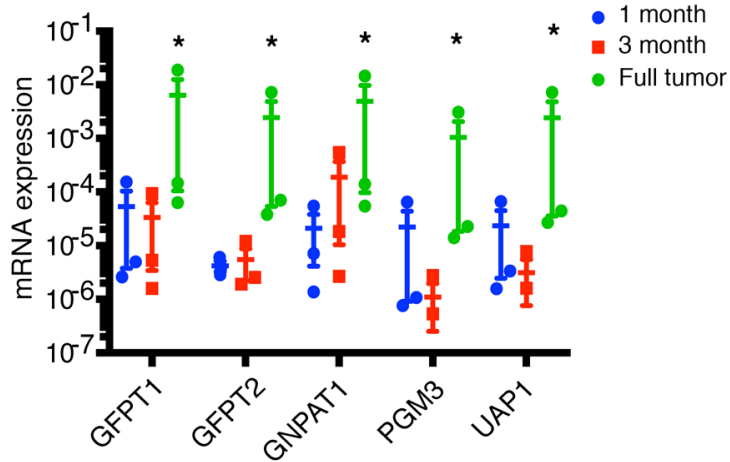

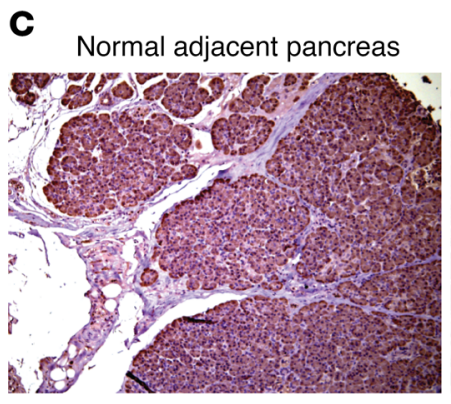

PDAC T2NOMO

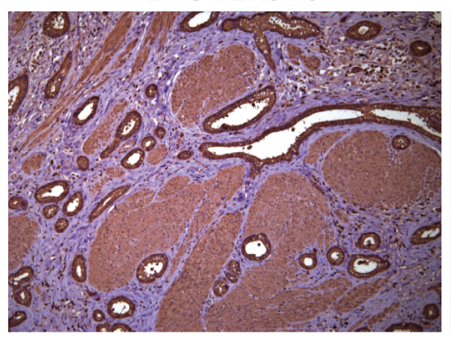

Cancer adjacent pancreas

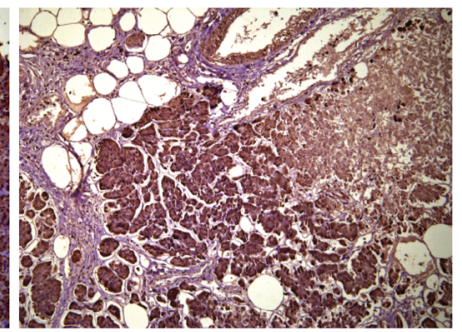

PDAC T3NOMO

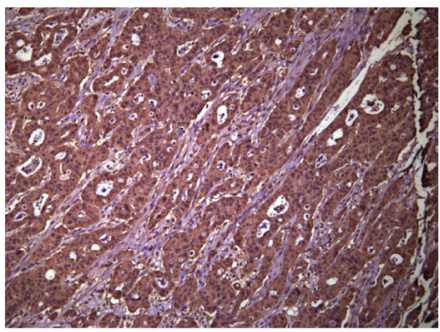

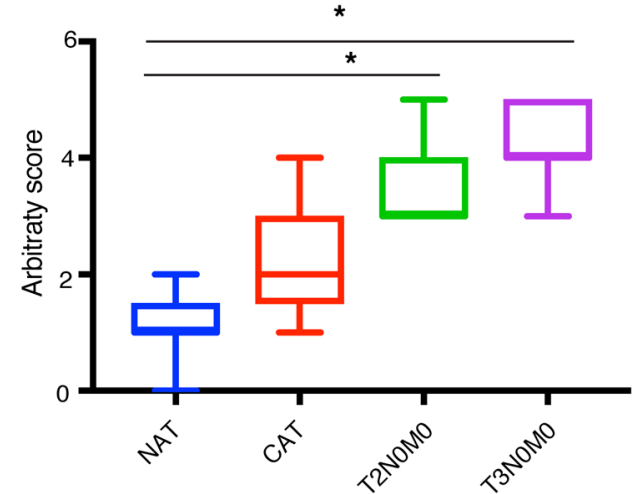

D
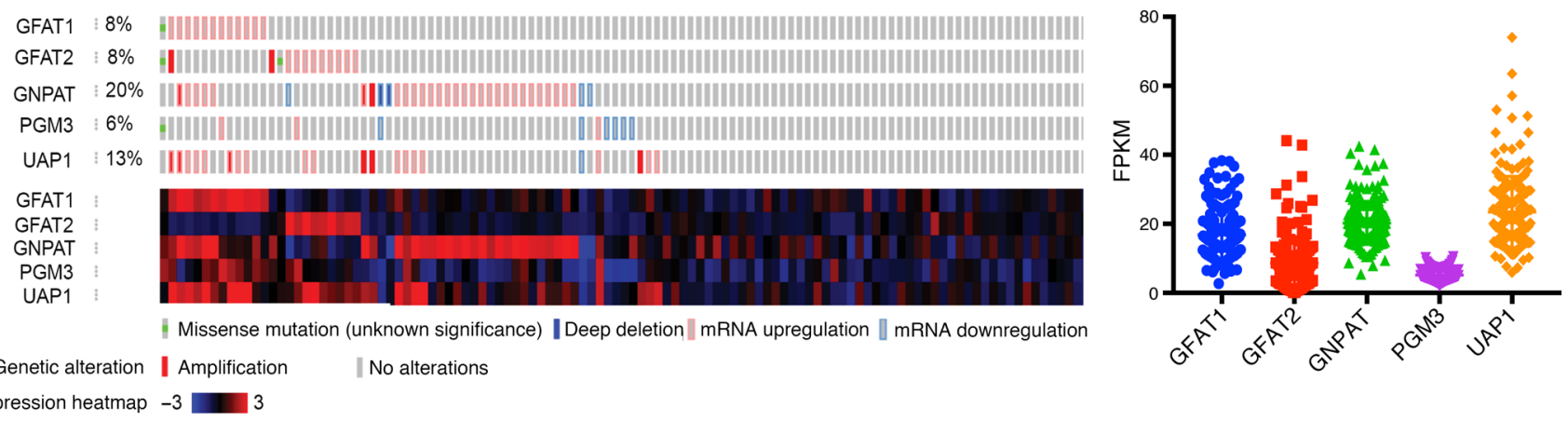

Expression heatmap -3

Figure 1. Hexoamine biosynthesis pathway is activated in PDAC and chronic pancreatitis. Enzymes in the HBP are overexpressed in pancreatitis (A) as well as in pancreatic cancer mouse model KPC. Expression of enzymes increased as the tumor progressed (B). In tumor tissue microarray of PDAC patients, GFAT1, the rate-limiting enzyme of HBP, was overexpressed. The increased expression correlated with advanced grade of the tumor (C).

Original magnification, $\times 20$. The microarray contained $2-3$ samples of each disease stage. According to cBioPortal, a large number of patient cohorts in TCGA showed alterations in the genes of HBP $(n=176)$. Fragments per kilobase of transcript per million (FPKM) mapped reads correlate with relative expression of a transcript proportional to the number of CDNA fragments that originate from it (D). All gene expression studies with quantitative PCR (qPCR) were done using 3 independent biological replicates. Statistical significance was determined by using 2-tailed Student's $t$ test. Error bars represent mean \pm SEM. ${ }^{*} P<0.05$. 
A
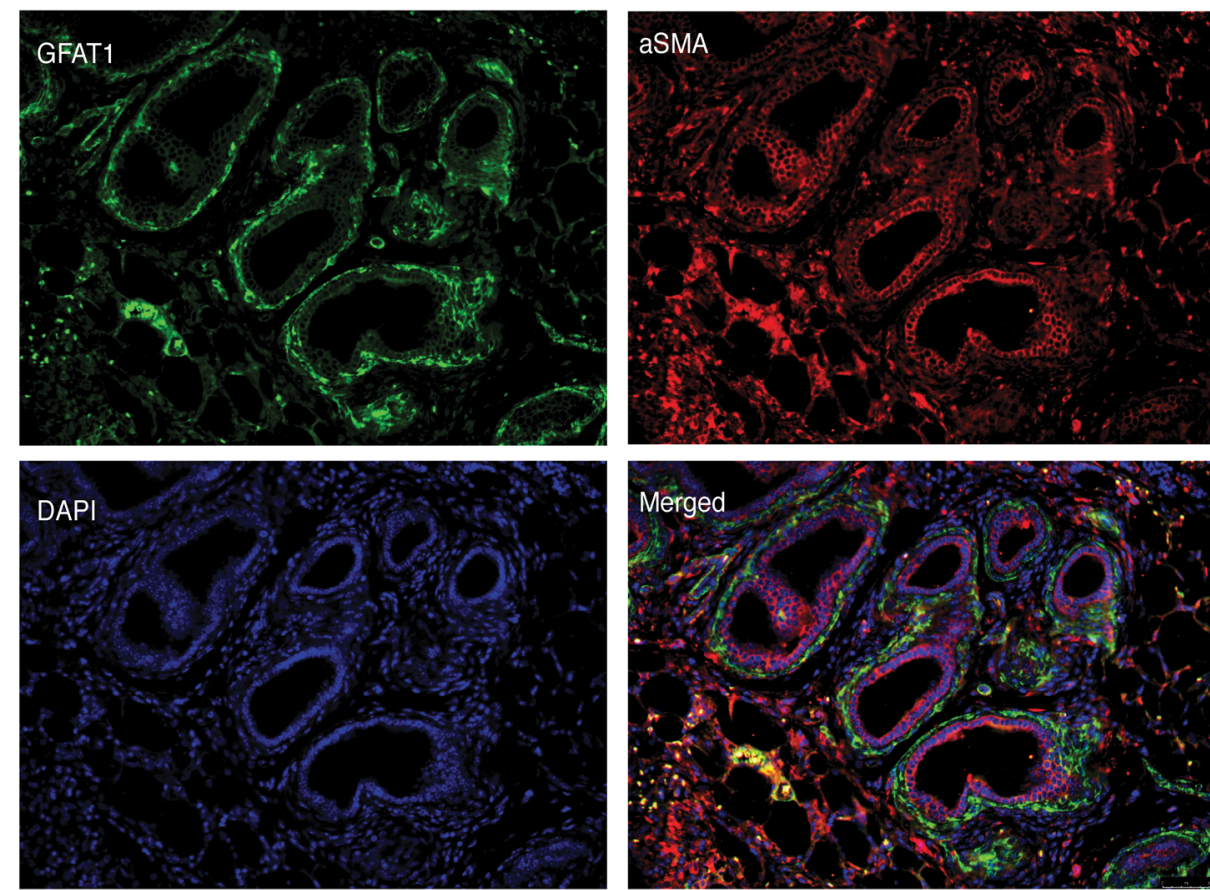

B
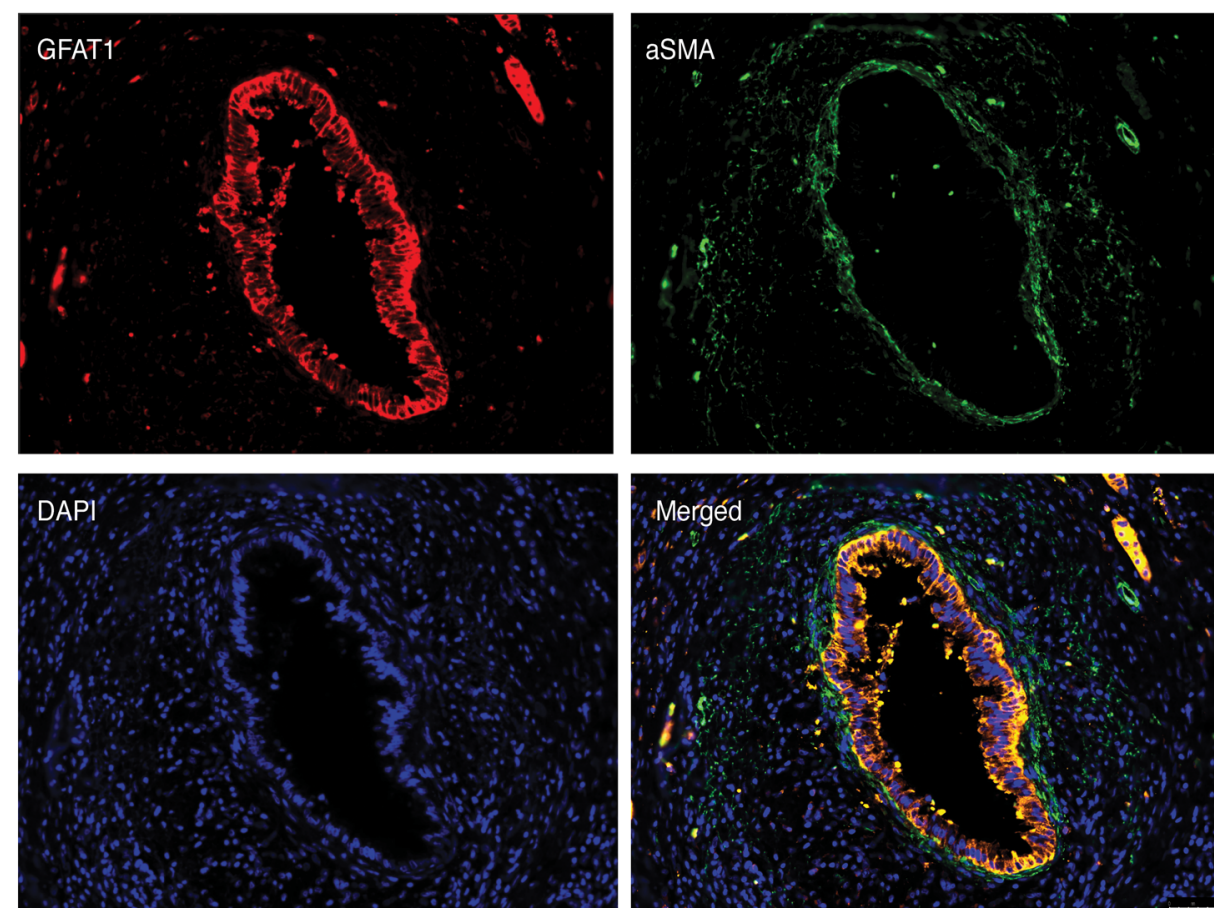

Figure 2. GFAT1 expression is shown predominantly in the ductal cells in a pancreatic tumor. (A and B) GFAT1 expression was shown predominantly in the ductal cells, as seen in tumors from KPC mice (A) or patient tumor tissue (B). Photographs are representative of 3 patient samples and 10 fields per sample. Original magnification, $\times 20$.

pancreatic cancer cells (Figure 3B). This observation was further validated in the pancreatic cancer cell line L3.6PL (Supplemental Figure 1B). These observations indicated that DON suppressed self-renewal ability of pancreatic cancer cells. Our previously published data showed that DON affected tumor cell prolifer- ation (33). Our current study showed that treatment with DON decreased viability of primary KPC cells while it did not have any effect on the viability of primary CAFs in vitro (Figure $3 \mathrm{C}$ ), indicating that within a tumor, DON had differential effects on the cellular components.

Since HBP affects the activity of OGT, which in turn is instrumental in regulating the metastatic property of cancer cells (41-43), we next evaluated the effect of blocking HBP on invasion and migration. Treatment with DON as well as GFAT siRNA decreased the invasiveness of S2VP10 cells when evaluated in a Boyden chamber assay (Figure 3D). Treatment with DON further decreased migration of pancreatic cancer cell lines when evaluated in real-time via electric cell surface impedance sensing (ECIS) (Figure 3E), further showing that DON suppressed invasiveness and metastatic potential of aggressive pancreatic cancer cells (S2VP10).

Inhibition of GFAT1 or glutamine utilization by DON resulted in regressed tumors and decreased metastasis in animals. To study whether DON was efficacious in vivo, we implanted metastatic pancreatic cell line S2VP10 subcutaneously in the flanks of athymic nude mice. Treatment with DON $(1 \mathrm{mg} / \mathrm{kg} / 5$ days a week) decreased tumor progression (Figure 4A) as well as end-of-study tumor weight and volume (Figure 4, B and $C$ ). In addition, treatment with DON decreased $\mathrm{Ki}-67^{+}$cells in the tumor, indicating a loss in proliferative pancreatic cancer cells (Figure 4D and Supplemental Figure 2A).

To test the efficacy of inhibition of glutamine utilization on metastasis, we implanted KPC and CAF cells orthotopically in the pancreas of $\mathrm{C} 57 \mathrm{BL} / 6$ mice at a ratio of $1: 9$. DON $(1 \mathrm{mg} / \mathrm{kg} / 3$ days a week) was administered for 30 days. Metastatic spread to local and distant tissues was documented following necropsy. DON significantly decreased metastatic spread and increased necrosis (Table 1 and Supplemental Figure 2B). In addition, DON significantly decreased the number of circulating tumor cells in this model (Figure $4 \mathrm{E}$ and Supplemental Figure 2C).

To rule out off-target effects of small molecule DON, we next constructed a tet-driven shGFAT1 and transfected metastatic 
A
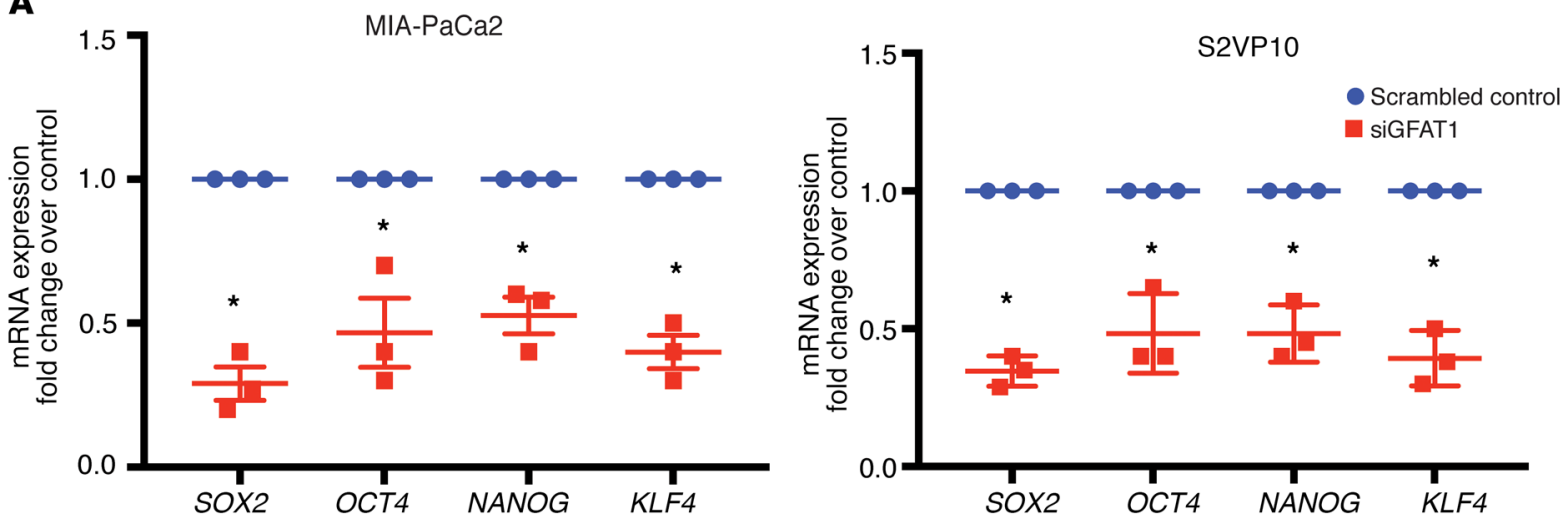

B

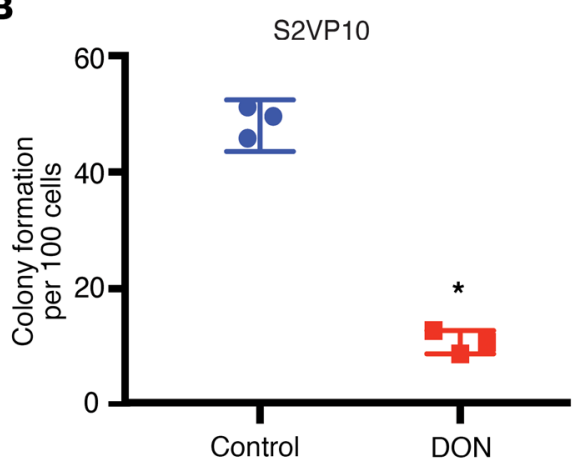

D

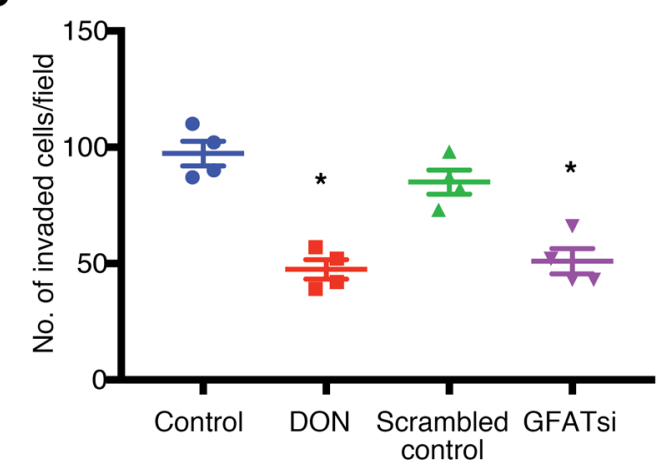

C
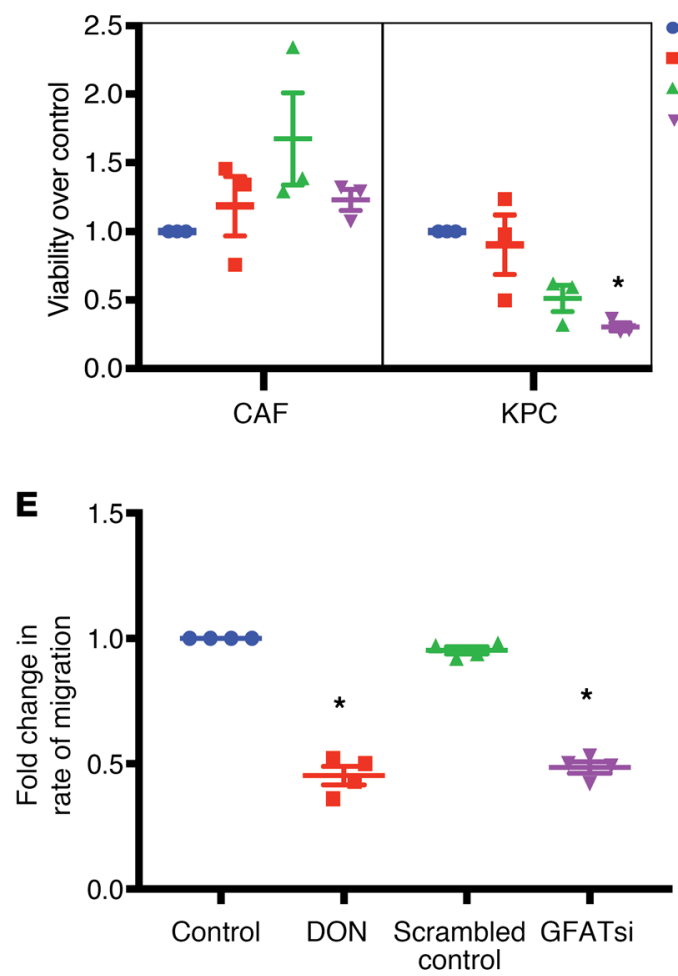

Figure 3. GFAT1 regulates self-renewal and invasion in PDAC. GFAT1 inhibition by siRNA decreased expression of self-renewal genes in pancreatic cancer cell lines MIA-PACA2 and S2VP10 (A). Treatment with glutamine analog DON at $50 \mu \mathrm{M}$ decreased colony formation in S2VP10 cells (B), indicating a loss of clonogenicity. Tumor epithelial cells (KPC) were more vulnerable to treatment with DON compared with CAF cells (C). Lethal concentration of $50 \%$ ( $\left.\mathrm{LC}_{50}\right)$ of DON for KPC cells was calculated to be $72 \mu \mathrm{M}$. At a nonlethal dose of $50 \mu \mathrm{M}$, treatment with DON decreased invasion (D) as evaluated by Boyden chamber assay as well as migration of pancreatic cancer cells S2VP10 as tested by ECIS (E). All gene expression studies with qPCR were done with 3 independent biological replicates. Student's $t$ test (parametric, 2 tailed) was used for statistical analysis, and the data are represented as mean \pm SEM. ${ }^{*} P<0.05$.

pancreatic cancer cell line S2VP10 to generate a tet-shGFAT1 cell line. These cells were implanted orthotopically in athymic nude mice. Ten days after implantation, GFAT1 expression was turned off by adding tetracycline to the chow. Animals were followed for an additional 30 days. As seen with DON, there was a significant reduction in end-of-study tumor volume (Figure $4 \mathrm{~F}$ and Supplemental Figure 2, D and E)

Treatment with DON modulates ECM in PDAC. Since HBP is responsible for synthesis of UDP-GlcNAc, which is also the substrate for HA, a major ECM component in pancreatic tumors, we next evaluated the effect of DON on ECM components of pan- creatic tumors. To study this, KPC001 and CAF cells (1:9 ratio) were implanted in the pancreas of C57BL/6 mice and treated with DON ( $1 \mathrm{mg} / \mathrm{kg} / 3$ days a week). Our studies showed that animals treated with DON had lower HA (Figure 5A and Supplemental Figure 3A) as well as lower collagen (Figure 5B and Supplemental Figure 3B). To study whether this decrease in HA and collagen was due to decreased gene transcription, we next studied the expression of genes involved in their synthesis. Our study showed that expression of HA synthase 1 (Has1) by the KPC tumor cells was significantly downregulated in the DON-treated group (Figure $5 \mathrm{C}$ ). Since other ECM components are equally synthesized by 
A

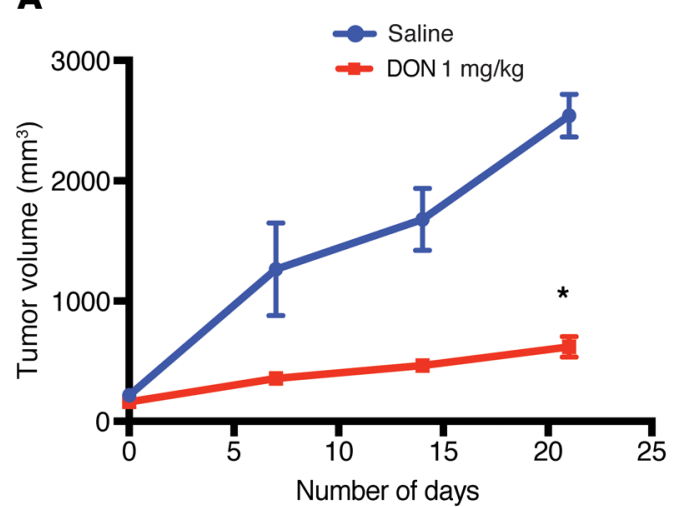

C

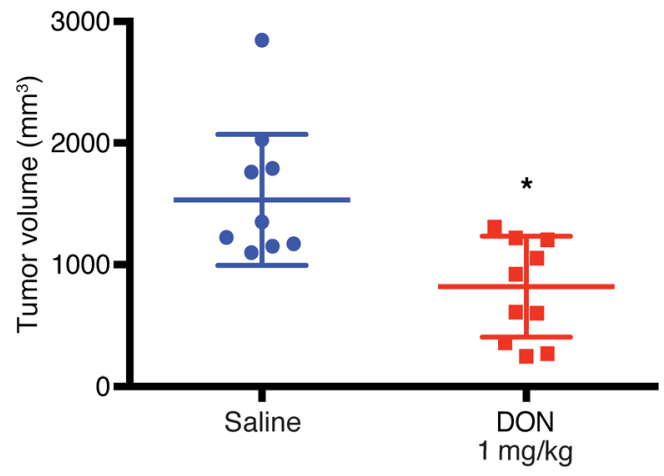

E

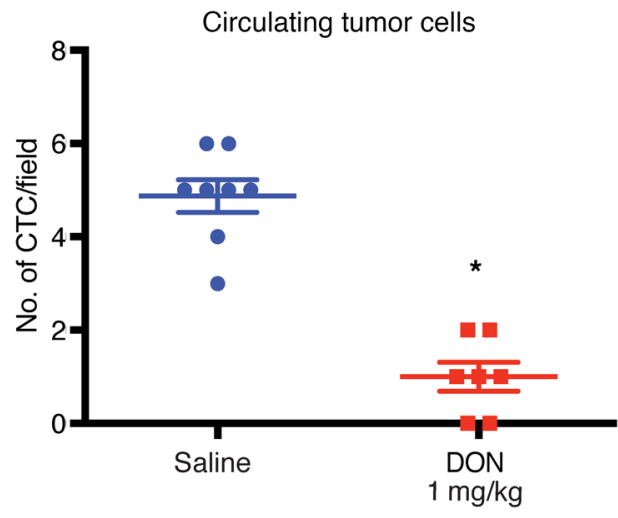

B

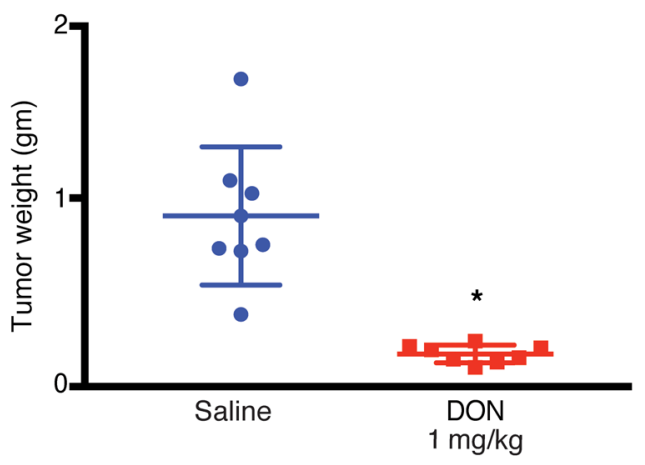

D
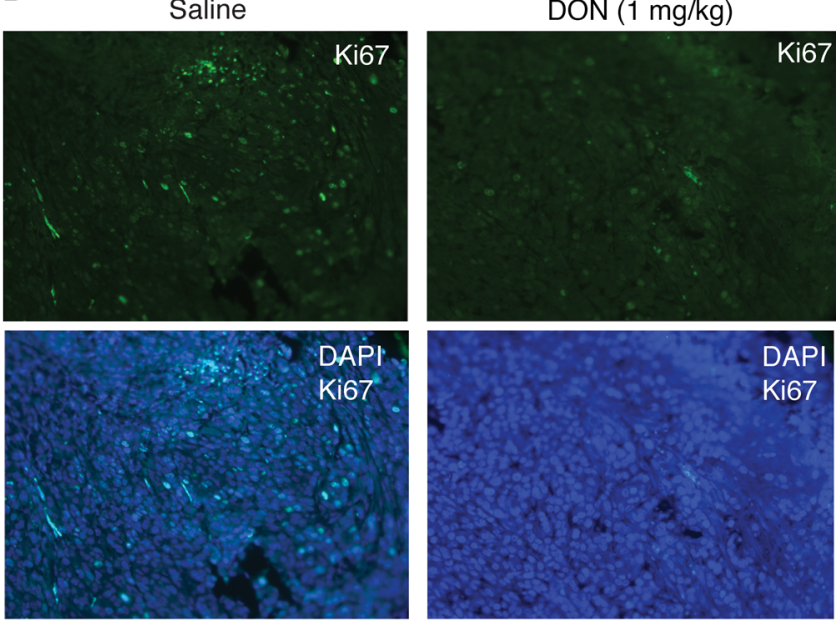

$\mathbf{F}$

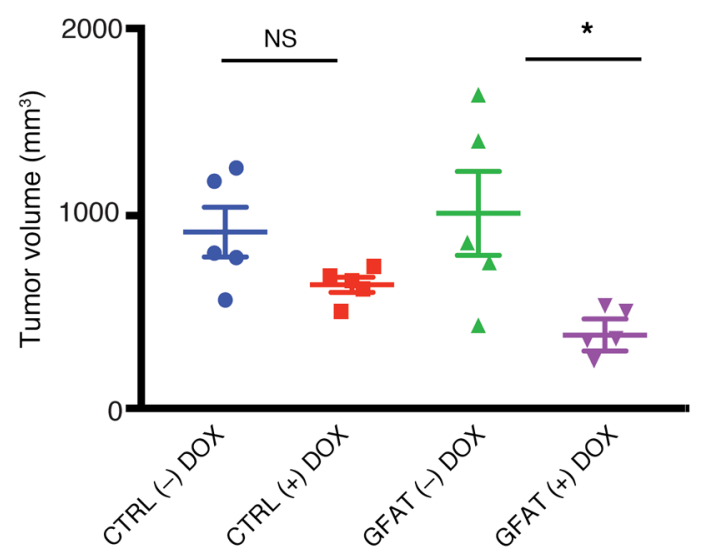

Figure 4. Inhibition of GFAT1 has antitumor effect in vivo. Treatment with $1 \mathrm{mg} / \mathrm{kg} / 5$ days a week of DON decreased tumor progression in subcutaneous implantation of pancreatic cancer cells S2VP10 in athymic nude mice $(n=10)(\mathbf{A})$. End point tumor weight $(\mathbf{B})$ and volume (C) were significantly decreased following treatment. Ki67 staining showed decrease in the number of proliferating cells. Original magnification $\times 10$ (D). Orthotopically implanted KPC:CAF in the pancreas of C57BL/6 were treated with DON (1 mg/kg/3 days a week) for 4 weeks. Treatment group showed decreased metastatic spread (Table 1$)$. Consistent with this, DON-treated animals had fewer circulating tumor cells in their blood (E). To confirm that antitumor effects were due to GFAT1/2 inhibition by DON, a tet-inducible shGFAT1 cell line was developed and implanted orthotopically in athymic nude mice. As observed with DON, upon induction of shGFAT1 with doxycycline, the tumor volumes of the animals were significantly smaller compared with the those with no doxycycline tumors (F). Student's $t$ test, nonparametric (Mann-Whitney $U$ test), was used to determine statistical significance, and data are represented as mean \pm SEM. ${ }^{*} P<$ 0.05. $n=10$ mice for all groups. 
Table 1. Effect of DON (1 mg/kg) on metastatic potential of pancreatic tumors

\begin{tabular}{lcc} 
Abdominal wall & $\begin{array}{c}\text { Control mets } \\
\text { in site/no. of animals }\end{array}$ & $\begin{array}{c}\text { DON }(1 \mathrm{mg} / \mathbf{k g}) \text { mets } \\
\text { in site/no. of animals }\end{array}$ \\
\hline Peritoneum & $5 / 10$ & $0 / 10$ \\
Spleen & $3 / 10$ & $0 / 10$ \\
Liver & $2 / 10$ & $0 / 10$ \\
& $6 / 10$ & $0 / 10$
\end{tabular}

tumor epithelial cells and stromal fibroblasts, we next cocultured KPC001 (murine primary tumor cells) and CAF cells and treated them as indicated in Supplemental Figure 3C. Our study showed that, while there was a significant decrease in the number of collagen synthesis genes along with expression of genes involved in other structural components of ECM (Figure 5D), there was a more profound effect on the ECM-remodeling proteases (Figure $5 \mathrm{E})$. This indicated that there was extensive ECM remodeling in the pancreatic tumor microenvironment. In addition, there was also a significant alteration of expression of a number of cell-cell and cell-ECM adhesion molecules (Supplemental Figure 3D).

It is well known that the tumor and stromal cells remodel the ECM, not only via synthesis of basement membrane constituents, but also through cytokine secretion. To evaluate this, we next set up the coculture as described in Supplemental Figure 3C and estimated the secreted cytokine profile using a cytometric bead array. Upon coculture in a Transwell, in which CAFs were treated with DON, IFN- $\gamma$ and IL- 6 were observed to be significantly altered by DON treatment, while only IL-27 showed a significant decrease when only KPC cells were treated with DON in the coculture (Figure 5F). Treatment of tumor cells (KPC) with DON completely abolished the secretion of IL-27 in the coculture, while treatment of CAF cells with DON did not significantly change the secretion of this cytokine. In CAFs, IL-6 secretion was substantially inhibited upon treatment, while changes in Mcp1 and IL-27 were not statistically significant. Since IL-6 and IL-27 are both protumor cytokines, their downregulation by DON indicated a profound antitumor activity of this compound. Similarly, IFN- $\gamma$ plays a role in activation of M1 macrophages as well as infiltration of $\mathrm{T}$ cells in the tumor, eliciting a tumor tissuedisruptive effect (44). These changes following treatment with DON indicated that DON played an antitumor role by affecting the tumor microenvironment of the pancreatic tumor (45).

Inhibition of GFAT1/HBP affects immune landscape in PDAC. It is well known that remodeling the ECM in a tumor affects its immune landscape (22). Based on the change in the secretion profile of cytokines from the tumor cells and stromal cells upon treatment with DON, it seemed likely that this would significantly affect the infiltration and function of immune cells in the pancreatic tumor. Macrophage density has been correlated with overall survival in pancreatic cancer patients (46). Our results showed that treatment with DON resulted in an increase in the activated macrophage population, as seen by CD68 staining (Figure 6, A and B). Since our previous results showed that treatment of pancreatic tumor cells with DON (KPCs or CAFs) increased IFN- $\gamma$ (Figure 5F), it is possible that the increased
$\mathrm{CD} 68^{+}$macrophage population within DON-treated tumors is a direct consequence of that event.

Our analysis of the tumor tissue further showed that there was a significant increase in the intratumoral $\mathrm{CD}^{+} \mathrm{T}$ cells (Figure 6, C and D). Increased infiltration of $\mathrm{T}$ cells is associated with better prognosis in pancreatic cancer. This indicated that DON was modulating the immune milieu of the pancreatic tumors by increasing the CD $68^{+}$ macrophages and promoting increased infiltration of $\mathrm{CD}^{+} \mathrm{T}$ cells, thereby reversing the immune-suppressive microenvironment.

To study whether DON was still an effective antitumor compound in the absence of $\mathrm{CD}^{+} \mathrm{T}$ cells, we next implanted KPCOO1 and CAF cells orthotopically in the pancreas of CD8-KO mice in a ratio of 1:9. The tumors were allowed to grow for 2 weeks before they were randomized to DON $(0.5 \mathrm{mg} / \mathrm{kg} / 3$ days a week) treatment or control groups. Treatment was continued for 30 days, and survival analysis was performed. Our results showed that survival of DON-treated animals in the CD8-KO mice was not different from the untreated animals (Figure 6E). Further, necropsy of animals across both groups showed no differences in tumor volume (Figure 6F). These results suggested that efficacy of DON in an immune-competent syngeneic pancreatic cancer model was largely dependent on $\mathrm{CD}^{+} \mathrm{T}$ cells.

Inhibition of GFAT1/glutamine utilization sensitizes to anti-PD1. Increased $\mathrm{CD} 8^{+} \mathrm{T}$ cells within a tumor have been associated with increased sensitivity to immune therapy in a number of cancers (47). Since treatment with DON alone resulted in modulating the ECM, altered cytokine secretion, and increased activated CD68 ${ }^{+}$ macrophages as well as cytotoxic $\mathrm{CD}^{+} \mathrm{T}$ cell infiltration in the pancreatic tumor, we next evaluated whether treatment with DON also made pancreatic cancer susceptible and sensitive to immune therapy. To study this, KPC001 and CAF cells were implanted orthotopically in the pancreas of $\mathrm{C} 57 \mathrm{BL} / 6$ mice at a ratio of $1: 9$. The tumors were allowed to grow for 14 days, after which animals were randomized into 4 groups: control/isotype $\mathrm{Ab}, \mathrm{DON}(0.5 \mathrm{mg} / \mathrm{kg})$, anti-PD1 $\mathrm{Ab}(100 \mu \mathrm{g} / 3$ injections), and anti-PD1+DON (0.5 mg/ $\mathrm{kg}$ ). Treatment was continued for 1 month, after which the animals were sacrificed. Our results showed a profound effect on the tumor weight (Figure 7A) and tumor volume (Figure 7B) with the combination of DON and anti-PD1. Additionally, the combination of DON and anti-PD1 resulted in a better survival advantage compared with either group alone (Figure 7C). Further, the combination resulted in a decrease of PDL1 expression in the tumor, indicating that this immune-evasive property of the tumor was overcome by DON (Figure 7D). Assessment of the ECM components following treatment with DON and anti-PD1 Ab showed that DON alone as well as in combination with anti-PD1 decreased HA and collagen (Figure 7E and Supplemental Figure 4, C, D, and E). Additionally, we also analyzed the expression of other checkpoint inhibitors in the tumor. Our results showed that, while expression of $\mathrm{B} 7-\mathrm{H} 3$ did not change with DON, expression of TIM3 and CTLA-4 were downregulated following treatment with DON (Supplemental Figure 5, A-C). We also analyzed the immune cells from the spleen of tumor-bearing mice. As seen from the tumor, DON-treated mice had increased infiltration of CD8+ T-cells as seen in Supplemental Figure 5G. Further characterization showed these $\mathrm{CD} 8^{+}$cells to have low PD1 expression, showing that they were not exhausted (Supplemental Figure 5, D-J). 
A
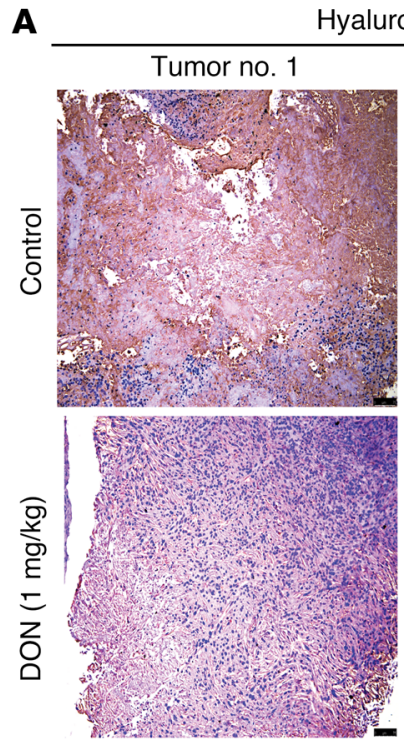

C

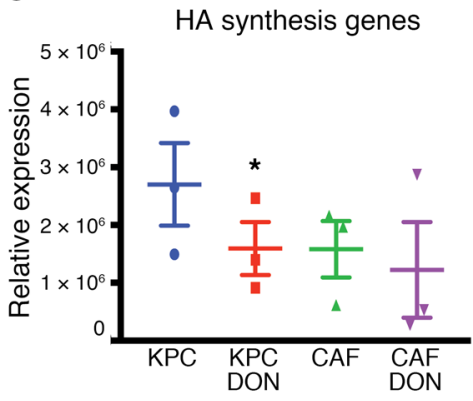

B Tumor no. 2
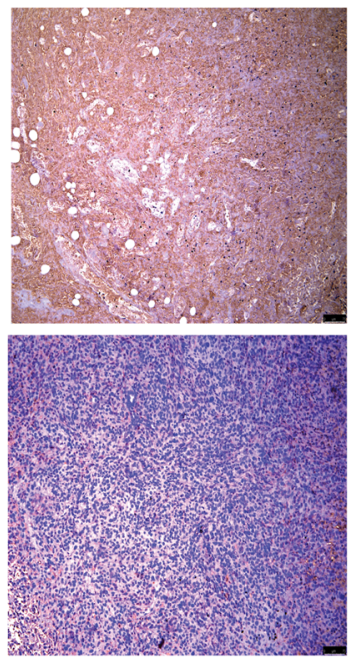

D
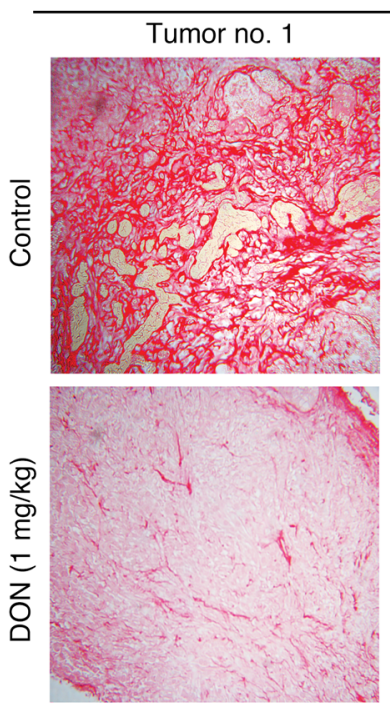

Collagen and other structural

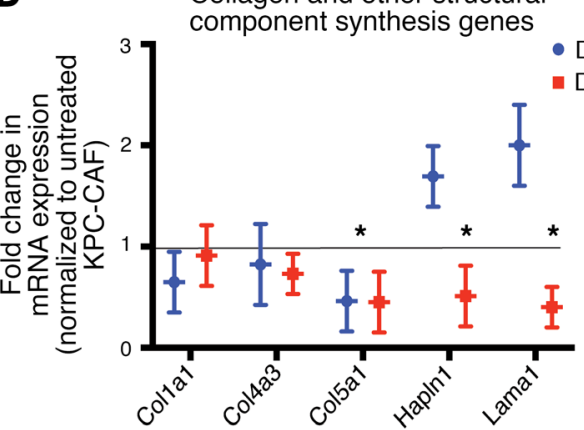
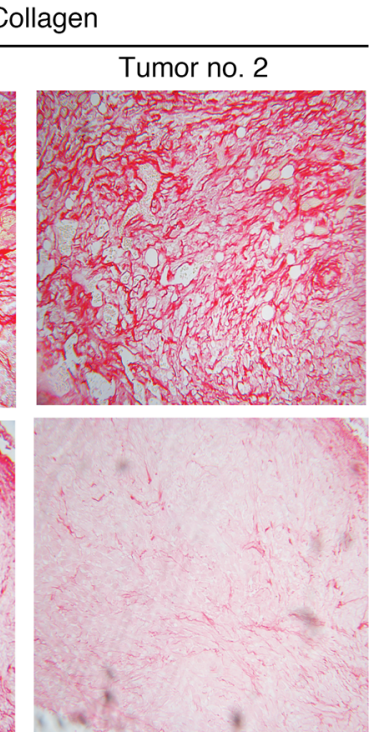

E

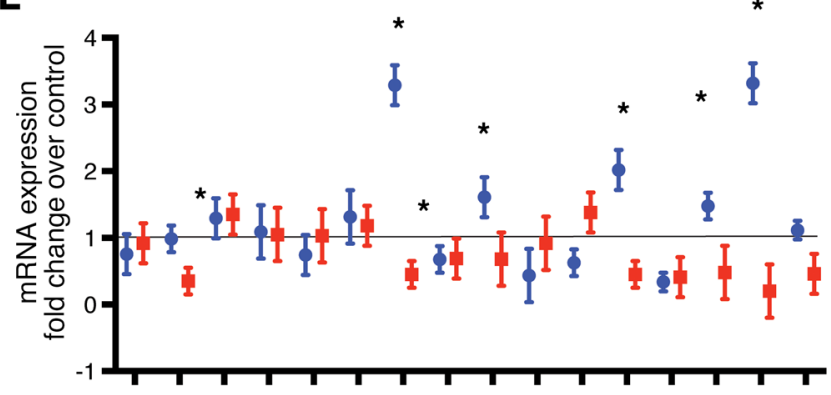

- DON CAF on KPC

- DON KPC ON CAF

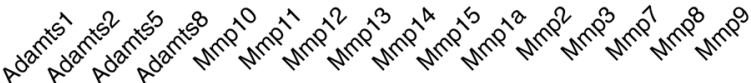

$\mathbf{F}$

Secreted cytokine analysis
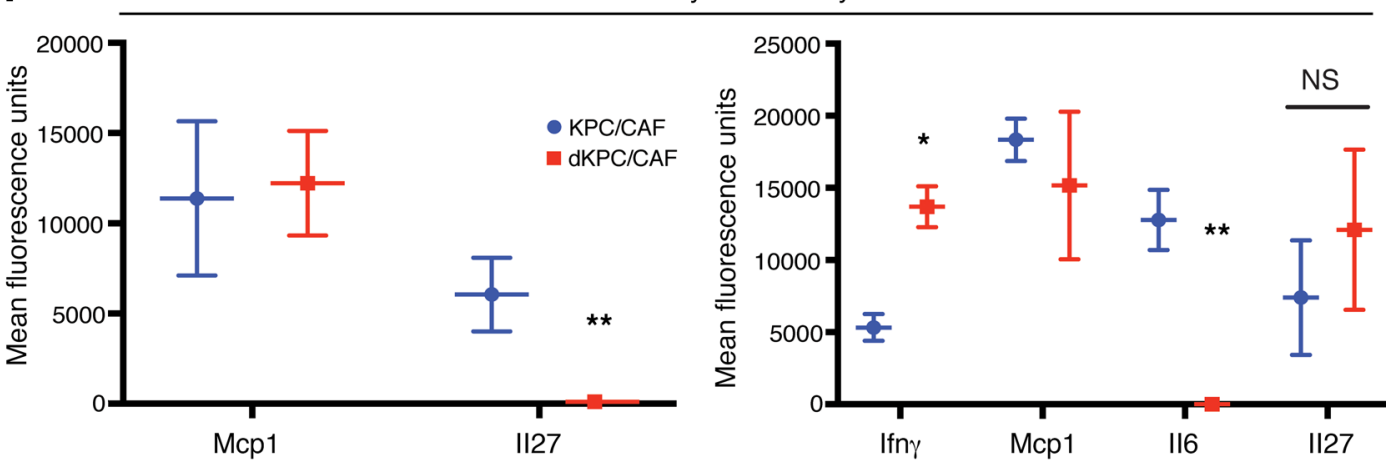
Figure 5. Treatment with DON remodeled ECM in orthotopic syngeneic mouse model. KPC and CAF cells were implanted orthotopically in the pancreas of C57BL/ 6 mice and treated with $1 \mathrm{mg} / \mathrm{kg}$ DON/3 days a week for 30 days. Treatment with DON significantly decreased HA (A) and collagen $(B)$ in the ECM of the tumor. Original magnification $\times 10$. Genes responsible for HA synthesis, such as HAS1, were decreased significantly in the KPC cells, but did not change in CAF cells (C). In a coculture experiment, treatment with DON $(50 \mu \mathrm{M})$ significantly decreased collagen synthesis genes in both KPC and CAFs, while other structural component genes such as Hapln1 and Lama1 were only decreased when DON-treated KPCs were cocultured with CAFs (D). Treatment with DON also changed expression of a number of ECM proteases significantly (E). Further, the secreted IL-27 was significantly decreased in DON-treated KPC cells, while IL-6 was significantly decreased in DON-treated CAF cells. Secretion of IFN- $\gamma$ was increased in CAFs upon treatment with DON (F). Each experiment was repeated 3 times, and the result is represented as mean \pm SEM. Student's $t$ test ( 2 tailed) was used for determining statistical significance. ${ }^{*} P<0.05$ and ${ }^{* *} P<0.02 . n=3$.

\section{Discussion}

Glutamine is one of the metabolites that is essential for growth and proliferation of cancer cells. It fuels a number of biosynthetic reactions in cancer cells $(48,49)$. Consistent with this, glutamineutilizing enzymes, such as GFAT1, are overexpressed in most cancers, including pancreatic cancer, as reported by our lab (Figures 1 and 2 ) and others $(50,51)$. Pancreatic cancer cells have been shown to utilize glutamine in a noncanonical fashion to support proliferation via redox homeostasis $(39,52-55)$. As glutamine metabolism is dispensable for nonmalignant cells, yet has a critical role in PDAC survival, it is an ideal candidate for therapeutic targeting.

Among glutamine-utilizing enzymes, GFAT1 is the ratelimiting enzyme for the HBP. This pathway is a shunt pathway of glycolysis that utilizes fructose- 6 phosphate from glycolysis and glutamine to synthesize UDP-GlcNAc, a nucleotide sugar that is essential for glycosylation reactions. Apart from $\mathrm{N}$-glycosylation and O-GlcNacylation, UDP-GlcNAc is also a substrate for HA synthesis. HA is a key component of the ECM, and therefore, inhibition of HBP is likely to affect the ECM in a tumor $(15,56-58)$. Thus, inhibition of GFAT1 effectively inhibits metabolic flux through this pathway and is likely to affect the glycosylation profile of the tumor cells as well as the components of the ECM. The ECM in a tumor actively drives tumor progression by providing a dynamic niche that regulates both mechanical and signaling abilities of a tumor cell (22). The ECM further plays a role in efficient infiltration of T cells in the pancreatic tumor. Thus, targeting glutamine utilization is likely to remodel the pancreatic cancer microenvironment and make tumors amenable to immune therapy. This makes DON, a glutamine analog, very well suited for evaluation against PDAC, as it is likely to have an antitumor effect (by directly targeting signaling pathways through its effect on glycosylation reactions) as well as an antistromal effect (by interfering with HA synthesis in the $\mathrm{ECM}$ ). Targeting just the microenvironment in pancreatic tumors, whether just cellular components (such as fibroblasts) or acellular components (such as ECM), has been controversial $(11,59,60)$. In fact, the stroma has been proposed to be a "restraining" mechanism of the host cells (13). However, preclinical studies from our lab as well as others have shown that the tumor microenvironment can be targeted only when the targeting agent can affect both tumor epithelial cells and stromal cells $(10,61,62)$. Thus, treatment with DON, which increases antitumor activity and remodels the ECM to promote infiltration of cytotoxic T cells in combination with anti-PD1 therapy, is a promising strategy for combatting pancreatic cancer.

Treatment with DON primarily affects the ECM components of the tumor and, as expected, decreases the noncellular components of the tumor. Our study showed that preventing utilization of glutamine (with DON treatment), and therefore inhibiting HBP, has a profound effect on the ECM composition of pancreatic tumors (Figure 5). Both collagen and HA in the ECM showed a decrease in the treated samples. Similarly, inhibition of GFAT in the tumors by tet-inducible shGFAT1 showed similar effects on collagen and HA content within the tumor (Supplemental Figure 2D). In addition, treatment of a KPC/CAF coculture with DON in vitro showed that there was extensive downregulation in the expression of ECM proteases such as MMP12, MMP7, and MMP8 (Figure 5). These proteases are involved in dynamic remodeling of the ECM during tumor progression, specifically regulating metastasis (63). Recent studies have shown that amino acid-mediated metabolic crosstalk between CAFs and tumor epithelial cells affects the biophysical as well as biological properties of the tumor (64). Inhibition of HBP with DON (or by shGFAT1) results in altered expression of the ECM proteases along with the synthesis of genes involved in ECM cell adhesion, indicating an extensive remodeling of the tumor microenvironment.

Treatment with DON also increased the infiltration of $\mathrm{CD} 68^{+}$ macrophages (Figure 6, A and B). This could be a consequence of increased Ifn- $\gamma$ (Figure $5 \mathrm{~F}$ ) in the tumor cells. CD $68^{+}$macrophages are considered to be "antitumor," as they secrete tumoricidal cytokines, such TNF- $\alpha$, IL-12, reactive nitrogen species, and oxygen intermediates. Additionally, $\mathrm{CD}^{+} 8^{+}$macrophages also promote infiltration of cytotoxic $\mathrm{CD} 8^{+} \mathrm{T}$ cells (44). Our results show that DON indeed promoted infiltration of $\mathrm{CD}^{+} \mathrm{T}$ cells in addition to increasing $\mathrm{CD}_{68}{ }^{+}$macrophages.

Apart from promoting tumor progression and metastasis, the ECM plays an active role in maintaining the immune landscape within a tumor. ECM regulates the migration of $\mathrm{T}$ cells toward the tumor cells by providing a 3D matrix as well as a chemokine gradient (22). Lack of infiltrating $\mathrm{T}$ cells is considered to be one of the prime reasons for the immune-evasive phenotype of pancreatic cancer. Among the small population that does infiltrate, the robust ECM prevents it from migrating to the tumor cells in order to execute their cytotoxic activity. Our studies show that DON profoundly increases infiltration of $\mathrm{CD}^{+}$cytotoxic $\mathrm{T}$ cells (Figure 6). In vitro, treatment with DON did not seem to affect T cell viability, but significantly increased T cell migration (Supplemental Figure 4B). Thus, remodeling of the ECM by DON actually promotes macrophage activation along with $\mathrm{T}$ cell infiltration and migration into the pancreatic microenvironment. Our experiments further show that the effect of DON in the immune-competent syngeneic mice was mediated via increased infiltration of $\mathrm{CD}^{+} \mathrm{T}$ cells, as this was lost in tumors implanted in CD8-KO animals (Figure 6, E and F). In these animals, the tumor burden did not decrease following DON treatment. Our studies further show that DON sensitizes tumors to anti-PD1 therapy (Figure 7, A and B). The presence of $\mathrm{CD} 8^{+} \mathrm{T}$ cells within 
A

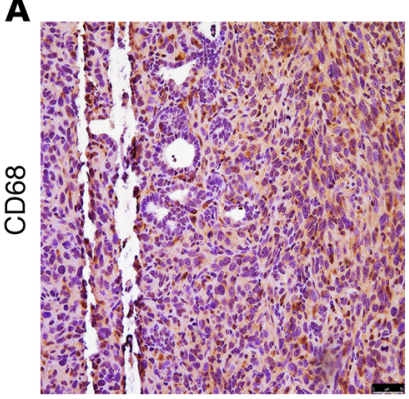

Control

C

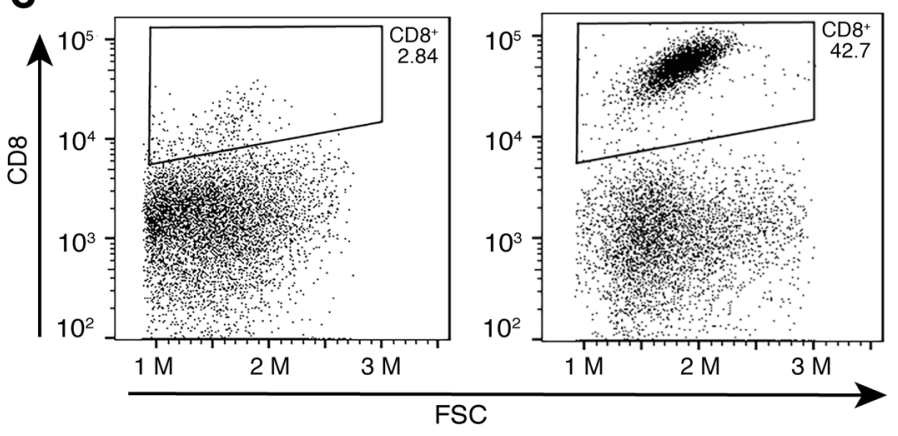

E

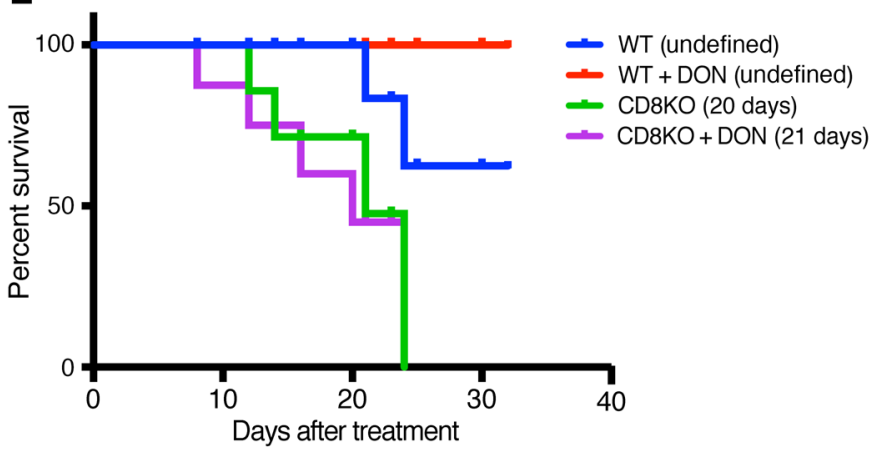

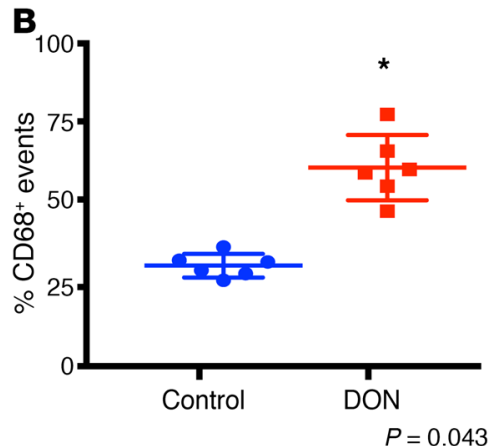

D

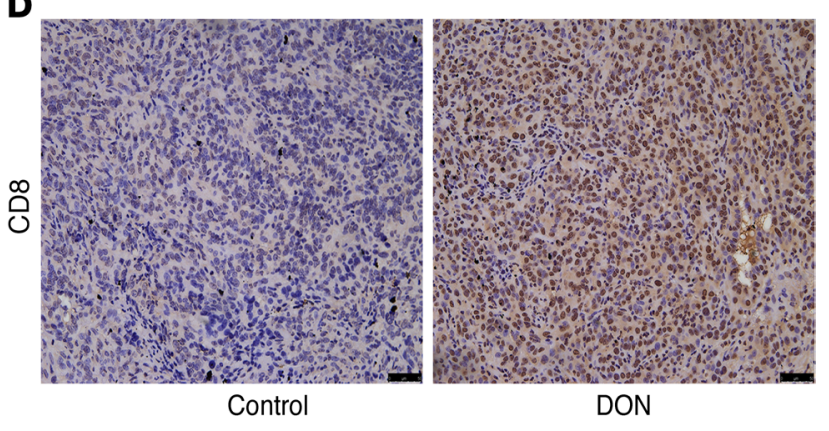

$\mathbf{F}$

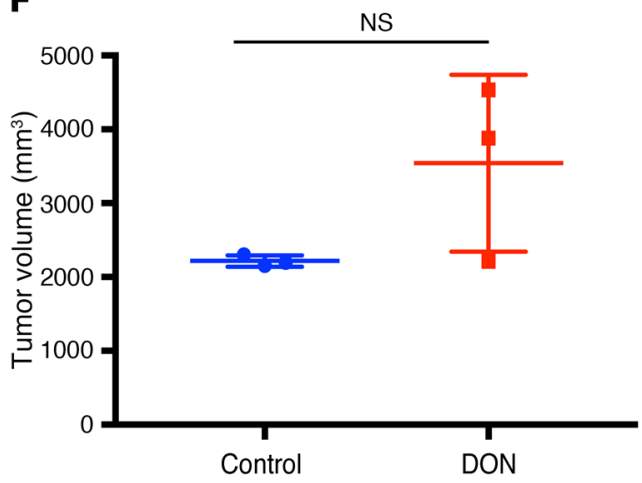

Figure 6. Treatment with DON altered immune profile in orthotopic syngeneic mouse model. In the orthotopic implantation of KPC and CAF in the pancreas of C57BL/6 mice, DON increased CD68+ macrophage populations (A and B). ${ }^{*} P<0.05$. Treatment with DON also increased intratumoral CD8 ${ }^{+}$infiltration, as seen by flow cytometry (C) and immunohistochemistry (D). Effect of DON on tumor and stroma in syngeneic model was CD8 mediated, as pancreatic tumors implanted in CD8-KO mice did not show improved survival $(\mathbf{E})$ or decrease in tumor volume $(n=3)(\mathbf{F})$ with DON. Original magnification, $\times 10(\mathbf{A}$ and $\mathbf{D})$.

the tumor improves response to anti-PD1 therapy (65). Thus, increased $\mathrm{CD} 8^{+} \mathrm{T}$ cells as a result of treatment with DON sensitize the pancreatic tumors to anti-PD1 therapy.

DON affects the tumor epithelial cells along with the ECM. Treatment with DON in a subcutaneous model of pancreatic cancer decreased tumor progression and also decreased tumor weight and volume (Figure 4). In an orthotopic model of pancreatic cancer, DON decreased metastatic spread of the tumor as well (Figure 4). This antimetastatic property of DON is of significance (Table 1), since DON also decreased ECM production in pancreatic tumors. As the remodeled and reduced ECM would have normally promoted tumors to metastasize to distant organs, the antitumor activity of DON prevented that. However, even though tumor cells responded to DON treatment, the stromal fibroblasts (or CAFs) were resistant to DON treatment, as seen by the viability assay done in vitro (Figure $3 \mathrm{C}$ ). This was an inter- esting observation, since athymic nude mice are immunocompromised, and if DON was facilitating its effect solely by altering the immune landscape, we would not have seen such an astounding effect on tumor regression in these mice. However, DON also had a profound effect on cancer cells, as it prevented glutamine utilization (since it is a glutamine analog). It is possible that in our in vivo experiment with the nude mice, in which the tumors lacked ECM and immune cells, the effects observed were purely due to the antitumor effect of DON. In the immune-competent mice, in which the tumors were coimplanted with CAFs that secreted a robust ECM, the ECM remodeling effects of DON promoted cytotoxic $\mathrm{T}$ cell infiltration and sensitized tumor to the anti-PD1 therapy. These data indicate that the combination effect of DON on inhibition of the cancer cells to metastasizing and the ECM remodeling to allow immune cell infiltration was responsible for the profound antitumoral effect of DON treatment. 
Converting "cold" pancreatic tumors that are unresponsive to immune therapy to "hot" and responsive tumors is a focus of pancreatic cancer research. Our studies show that DON, a glutamine analog, can be used for this. The antitumor effect of DON was observed earlier during phase 1 and 2 clinical trials in a number of cancers, including lung and colon cancer. Data from multiple trials demonstrated that DON was safe and could potentially be used as a single agent. These studies were conducted in the 1980s, and in $53 \%$ of patients, there were reports of a stable disease in colorectal cancer. Clinical studies of DON never reached phase III trials. The dose for DON used in these studies ranged from $50 \mathrm{mg} / \mathrm{m}^{2}$ to 480 $\mathrm{mg} / \mathrm{m}^{2}$. The lowest dose used $\left(50 \mathrm{mg} / \mathrm{m}^{2}\right)$ translates to roughly 6 $\mathrm{mg} / \mathrm{kg}$ in mice $(35,38,66)$. However, DON was never evaluated as a sensitizing agent for immune therapy in cancer. The potent effect of DON on ECM appeared to promote an increase in tumoricidal macrophages and an infiltration of cytotoxic $\mathrm{T}$ cells in our study. This implied that targeting glutamine utilization with DON in pancreatic cancer can be developed as a potential therapeutic option to sensitize the normally immune-resistant pancreatic cancer to anti-PD1 therapy.

Conclusion. PDAC is considered to be a highly immunosuppressive and heterogeneous neoplasm $(6,67)$. Despite improved knowledge regarding the genetic background of the tumor and better understanding of the tumor microenvironment, immune checkpoint inhibitor therapies (using CTLA-4, PD-1, PDL1) that have shown effect in other solid tumors have not been very successful in PDAC. Thus, novel therapeutic strategies that can make PDAC more amenable to immune therapy are urgently needed. In this context, our study is extremely timely, as it reveals how specific metabolic pathways can be targeted in cancer cells to overcome immune resistance and sensitize the tumors to anti-PD1-like therapy options that have worked extremely well in other cancers.

\section{Methods}

Cell lines, treatments, and reagents. SU.86.86 (CRL-1837) and MIAPaCa-2 (CRM-CRL-1420) were purchased from ATCC and were cultured according to the recommended conditions. Human pancreatic stellate cells (catalog 3830) were purchased from ScienCell. The primary KPC cell line was isolated from the tumor of a 5- to 6-monthold genetically engineered mouse model of KRAS ${ }^{\mathrm{G} 12 \mathrm{D}} \mathrm{P} 53^{\mathrm{R} 172 \mathrm{H}} \mathrm{Pdx}-1-\mathrm{Cre}$ (KPC) mice. The cells were isolated according to the protocol from our previous study (68). CAFs were isolated from KPC mice according to the protocol described by Sharon et al. (69). The purity of the fibroblasts was evaluated by flow cytometry using fibroblast surface protein (FSP) Ab and CK19 Ab. FSP ${ }^{+}$and CK19- populations were used for subsequent experiments. All the established cell lines were used from passages 5-20. S2-VP10 and L3.6pL cells were a gift from Masato Yamamoto's laboratory (University of Minnesota, Minneapolis, Minnesota, USA). Pancreatic stellate cells from mouse pancreas were isolated according to the protocol as described by Apte et al. (70). SU.86.86 and S2-VP10 cells were grown in RPMI 1640 (Gibco, Thermo Fisher Scientific) containing 10\% FBS and 1\% penicillin/streptomycin (Gibco, Thermo Fisher Scientific). MIA PaCa-2, KPCs, and CAFs were grown in DMEM high-glucose containing 10\% FBS and 1\% penicillin/streptomycin. All cell lines were routinely tested for mycoplasma and STR profiles (ATCC). DON (D2141) was purchased from Sigma-Aldrich and was used at a dose of $50 \mu \mathrm{M}$ for in vitro experiments.
Colony forming assay. S2-VP10 and L3.6PL cells were pretreated with DON for 24 hours. Cells were counted and plated at a density of $10 \mathrm{~K}, 1 \mathrm{~K}$, and 100 cells per well in both treatment and control groups respectively. Colonies were counted after 4 days of plating. The results represented are from 1000 cells per well, but the results were similar across different dilutions.

Immunohistochemistry. Tissues were deparaffinized by heating at $56^{\circ} \mathrm{C}$ overnight and then hydrated by treating with Xylene (15 minutes, 2 times), $100 \%$ ethanol, $90 \%$ ethanol, and $70 \%$ ethanol (2 times) at 5 minutes each. The slides were then steamed with a $\mathrm{pH} 6$ reveal decloaker (Biocare Medical) for antigen retrieval and blocked in Dako serum blocker (Agilent Technology). Primary Ab was added overnight. Slides were washed $3 \times$ in PBS, secondary Abs (AF 488 A32723 and AF 555 A32794, Thermo Fisher Scientific) were diluted in Sniper (Biocare Medical), and slides were stained for 30 minutes at room temperature. Slides were then washed again $3 \times$ in PBS and mounted using ProLong Gold anti-fade with DAPI (Molecular Probe, P36935) for immunofluorescence staining. For immunohistochemistry, the cells were then treated with DAB (SK-4100) and hematoxylin (H-3404, Vector Laboratories). The stained slides were then dehydrated by dipping in $70 \%$ ethanol (2 times), 90\% ethanol, 100\% ethanol and finally in xylene (15 minutes, 2 times). Slides were then mounted with paramount and dried overnight and imaged by a bright-field microscope. GFPT1 Ab (14132-1-AP) was purchased from Proteintech and used at a dilution of 1:200. $\alpha$-SMA (catalog ab5694), CD4 (catalog ab183685), CD8 (catalog ab22378), and PDL1 (catalog ab80276) were purchased from Abcam and were used at dilutions of 1:200, 1:1000, 1:200, and 1:50, respectively. For CD4 and CD8 Ab citrate buffer, Agilent S1699842 was used for antigen retrieval. Ki-67 SP6 Ab was purchased from Thermo Fisher Scientific and was used at a dilution of 1:200.

Animal studies. Female athymic nude (nu/J) mice (Jackson Laboratory) between the ages of 4 and 6 weeks were used for in vivo experiments. For subcutaneous experiments, 500,000 S2VP10 cells were implanted in the right flank of athymic nude mice. Corning Matrigel Growth Factor Reduced (GFR) Basement Membrane Matrix and 1× PBS at a ratio of 1:1 were used as a suspension medium for the cells. Tumors were allowed to reach a size of $100 \mathrm{~mm}^{3}$, and then the treatment was started. The dose of DON given for each experiment is specified in the figure legends. At the end of the fourth week, mice were sacrificed and tumor weight and volume were noted. Tissues were flash frozen for further experiments.

Female C57BL/6 mice (Jackson Laboratory) between the ages of 4 and 6 weeks were used for orthotopic implantation of KPC and CAF cells. $1000 \mathrm{KPC}$ and $9000 \mathrm{CAFs}$ were implanted orthotopically in 100\% Corning Matrigel GFR Basement Membrane Matrix. Tumors were allowed to grow for 2 weeks, after which mice were randomized and treatment was started. DON was given at a dose of $1 \mathrm{mg} / \mathrm{kg} / 3$ days a week. Orthotopic tumors were harvested, dimensions were noted, and metastasis was evaluated. Blood for CTC isolation was collected via cardiac puncture, and blood from 3 mice per group was pooled for CTC.

For evaluating the combinatorial effect of DON and anti-PD1 Ab therapy on tumor growth, female C57BL/6 mice between the ages of 4 and 6 weeks were used. $1000 \mathrm{KPC}$ and $9000 \mathrm{CAFs}$ were implanted orthotopically in $100 \%$ Corning Matrigel GFR Basement Membrane Matrix. Tumors were allowed to grow for 2 weeks, after which mice were randomized and treatment was started. DON was given at a dose 
A
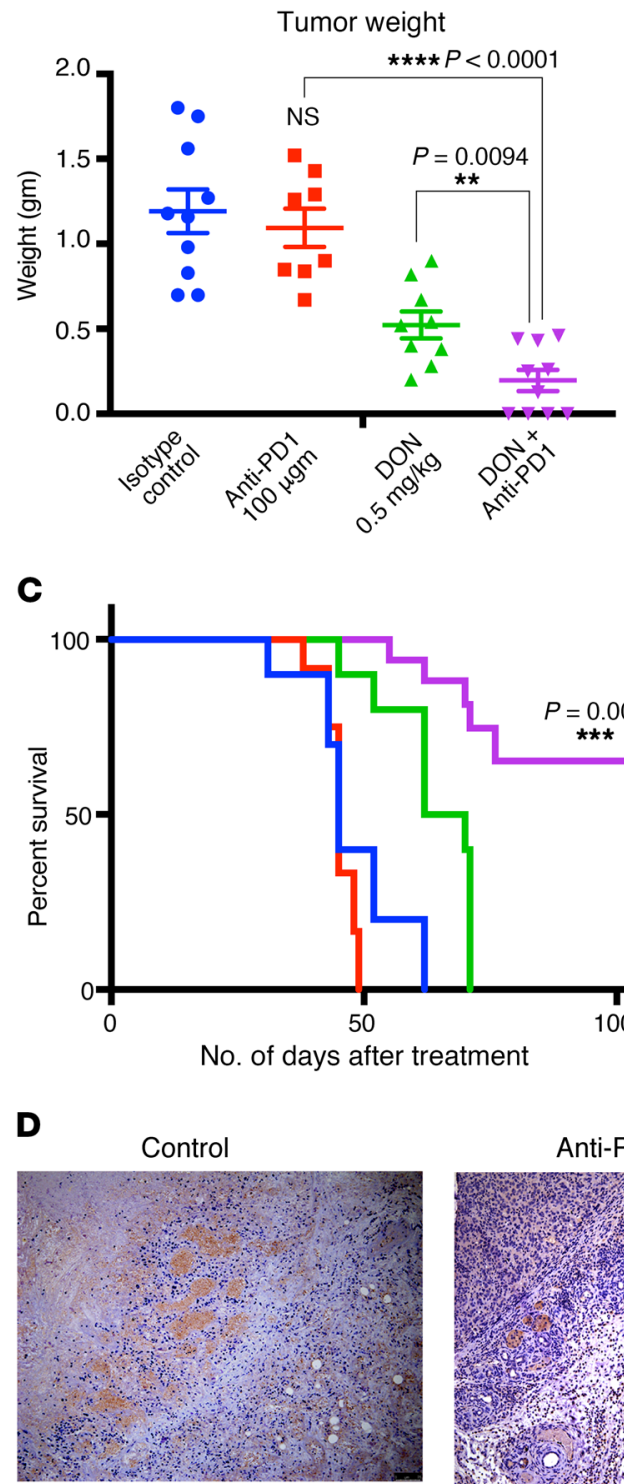

E
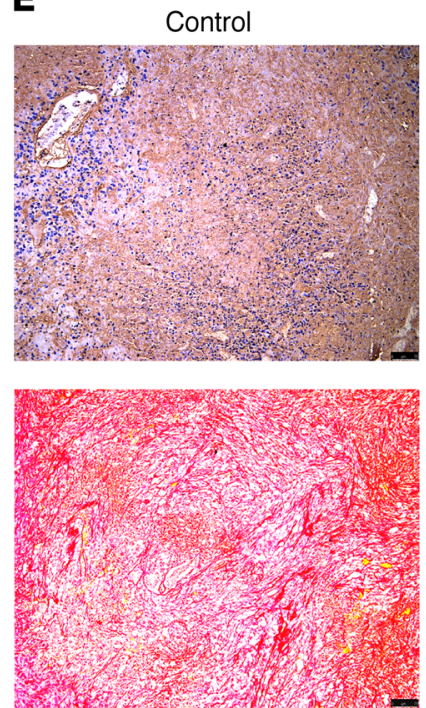

B

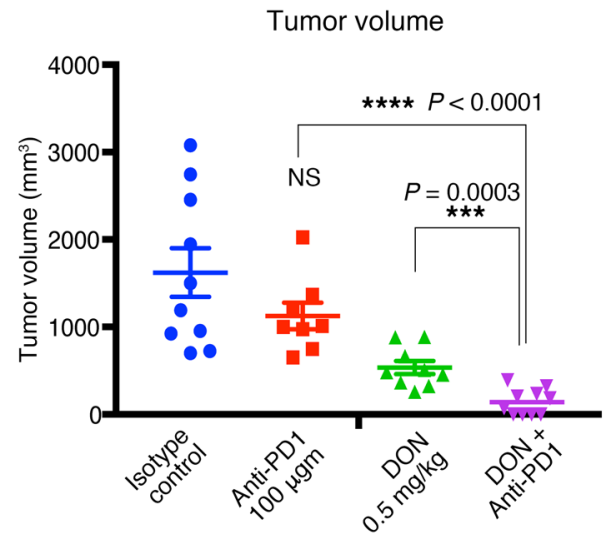

Figure 7. Treatment with DON sensitized PDAC to anti-PD1 Abs. Pancreatic tumor-bearing immunocompetent C57BL/6 mice were treated with isotype Ab, DON ( $0.5 \mathrm{mg} / \mathrm{kg} / 3$ days a week), anti-PD1 Ab (100 $\mu \mathrm{g} / 3$ times), and DON $(0.5 \mathrm{mg} / \mathrm{kg} / 3$ days a week)+anti-PD1 Ab (100 $\mu \mathrm{g} / 3$ times). Treatment with DON as well as with DON+anti-PD1 Ab showed significant decrease in tumor volume (A) as well as in tumor weight (B). Combination treatment also increased survival of tumor-bearing animals (C). DON decreased PDL1 expression, as seen by immunohistochemistry image (D). Treatment with DON decreased HA and collagen in the syngeneic tumors; however, the decrease of these ECM components in the combination group was marginal (E). The histological images for PDL1, HA, and collagen were acquired at $10 \times$ magnification. $n=10$ mice for each group. Result are represented as mean \pm SEM. One-way ANOVA was used for statistical analysis.
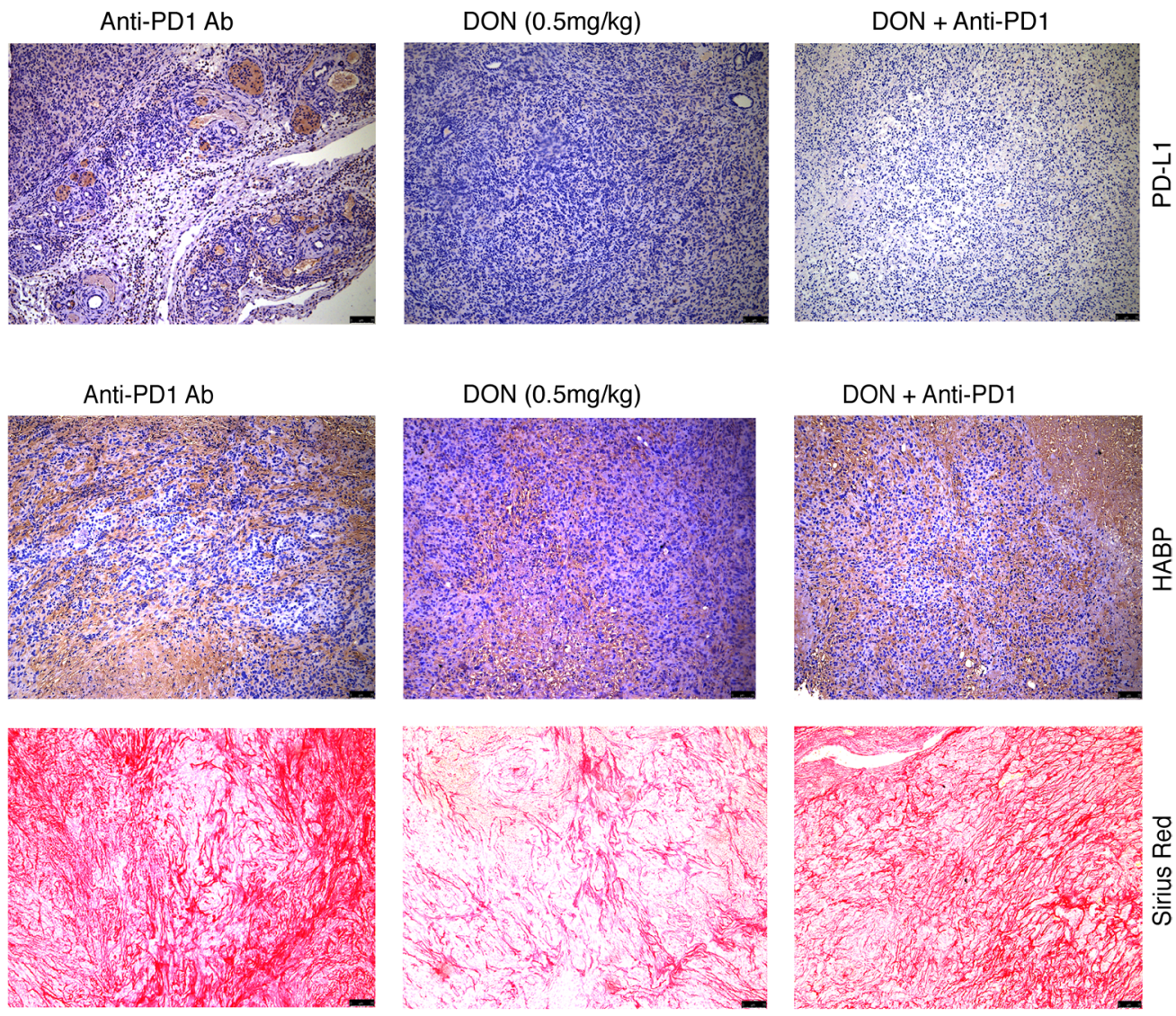
of $0.5 \mathrm{mg} / \mathrm{kg} / 3$ days a week, and anti-PD1 was given at a dose of 100 $\mu \mathrm{g}$ per mouse at days 15,17 , and 19 after starting DON treatment. DON was given for 4 weeks, after which mice were sacrificed, tumors were harvested, and metastasis was evaluated.

CD8-KO mice (B6.129S2-Cd8atm1Mak/J) were purchased from Jackson Laboratory. $1000 \mathrm{KPC}$ and $9000 \mathrm{CAF}$ cells were implanted orthotopically in 100\% Corning Matrigel GFR Basement Membrane Matrix in female CD8-KO mice between 4 and 6 weeks of age. Tumors were allowed to form for 2 weeks, after which the mice were randomized into control and treatment groups. DON was started at week 3 after surgery at a dose of $0.5 \mathrm{mg} / \mathrm{kg} / 3$ times a week. Mice were monitored daily for morbidity or mortality, and a survival curve was plotted.

Isolation of circulating tumor cells. Circulating tumor cells were isolated as described (71). Briefly, $0.5 \mathrm{ml}$ of blood was collected from animals in the control and DON-treated groups in EDTA-coated tubes. The blood was passed through the Circulogix FaCTChecker System and captured on the system's filter cartridges mounted on slides. Captured cells were stained with fluorescent-tagged Abs against CD45 and CK19. The $\mathrm{CD} 45^{-} \mathrm{CK} 19^{+}$fraction was quantitated from each group of animals.

Multiplexed cytometric bead array. KPC and CAF cells were pretreated with DON for 24 hours. 100,000 Cells were plated in Corning Transwell polycarbonate membrane cell culture inserts. The treatment groups were $\mathrm{KPC}(\mathrm{C})+\mathrm{CAF}(\mathrm{P})$, DON $\mathrm{KPC}(\mathrm{C})+-$ $\operatorname{CAF}(\mathrm{P}), \operatorname{DON} \operatorname{CAF}(\mathrm{C})+\mathrm{KPC}(\mathrm{P})$ DON KPC $(\mathrm{C})+\mathrm{DON}$ CAF $(\mathrm{P})$ and media, where $C$ indicates cells placed in the chamber and $P$ indicates cells on the plate. Cells were allowed to be in coculture for 24 hours. Conditioned media was collected after incubation. The LEGENDplex Mouse Inflammation Panel (BioLegend 740150) was used, and the experiment was performed according to the manufacturer's instructions.

RT-PCR array with coculture samples. KPC and CAF cells were plated and treated as described above for the multiplexed cytometric bead array assay. RNA was isolated from the bottom chamber via the TRIzol method, and cDNA was prepared using the high-capacity cDNA reverse transcription kit (catalog 4368814) from Applied Biosystems. The Mouse Extracellular Matric and Adhesion molecules RT ${ }^{2}$ PCR kit (PAMM-013Z) from QIAGEN was used,and the experiment was performed according to the manufacturer's instructions.

Flow cytometry for infiltrated immune cells. Tumor samples harvested from mice were placed in RPMI until they were ready to be processed. Tumors were minced into tiny pieces and then were digested with collagenase IV (Sigma Aldrich C7657) at $37^{\circ} \mathrm{C}$ for $1-3$ hours. After the tissue had been digested, they were passed through a $40 \mu \mathrm{m}$ nylon filter. The tissue was then spun at $500 \mathrm{~g}$ for $5 \mathrm{~min}$ utes and was then resuspended in $1 \mathrm{ml}$ of flow buffer (0.5\% BSA, 2 mM EDTA, 1\% penicillin-streptomycin in $500 \mathrm{~mL}$ PBS). Tubes were then spun again at $500 \mathrm{~g}$ for 5 minutes. $100 \mu$ l Cytofix-Cytoperm buffer was added to the pellet, and the cells were allowed to fix for an hour at room temperature. After 1 hour, cells were washed with 1 $\mathrm{ml}$ of FACS buffer and then centrifuged at $500 \mathrm{~g}$ for 5 minutes. The supernatant was then discarded, and the cells were stained with the surface and intercellular Abs for 40 minutes in the dark. After 40 minutes of staining, the cells were washed with $1 \mathrm{ml}$ of FACS buffer and then spun at $500 \mathrm{~g}$ for 5 minutes. Cells were then resuspended in $200 \mu \mathrm{l}$ of FACS buffer and were acquired. Abs used for T cell analysis were all from BioLegend: CD3 PE Dazzle (catalog 100348), CD4 PE Cy7 (catalog 10028), CD89AF-657, CD49b FITC (catalog
108906), CD25 AF-700 (catalog 102024), TCR $\gamma / \delta$ BV 510 (catalog 118131), FoxP3 PE (catalog 126404), IL-4 BV-711 (catalog 504133), IL-17 BV-421 (catalog 506926), TNF-a BV-650 (catalog 506333), IL10 APC/Cy7 (catalog 505036), and IFN- $\gamma$.

Boydon chamber invasion assay. 24-Well chamber inserts (Corning BioCoat) were hydrated in serum-free medium for 2-3 hours: the bottom of the chamber wells contained the attractant (10\% FBS contraining DMEM), and 50,000 cells were plated on the top of the insert. Twenty-four hours later, cells that did not invade were scrubbed from the top chamber via a cotton swab, and the invaded cells were fixed in methanol and stained with crystal violet. Invaded cells were counted by microscopy.

Migration assay. The migration assay was conducted by ECIS (Applied Biophysics) and performed as described in Banerjee et al. (72).

Tetracycline-inducible GFAT knockdown cell line. For generation of tet-inducible GFAT knockdown cell line, S2VP10 was selected as a parent cell line. shRNA oligos for GFPT1 were purchased from Integrated DNA Technologies (IDT), and the cell lines were developed according to the protocol described by Wiederschain et al. (73) and Wee et al. (74).

For further information see Supplemental Methods.

Statistics. All in vitro experiments were performed in 3 independent runs, and values were expressed as mean \pm SEM. Student's $t$ tests ( 2 tailed) were used to determine significance, and $P$ values of less than 0.05 were considered statistically significant. For experiments with more than 2 groups, 1-way ANOVA was performed using GraphPad Prism software.

Study approval. All animal studies were performed according to protocols approved by the University of Miami Department of Veterinary Research (DVR) and the Institutional Animal Care and Use Committee (IACUC). Deidentified patient TMAs were obtained from US Biomax Inc., with all tissue donors giving informed consent.

\section{Author contributions}

NSS, VKG, VTG, RH, BG, and AF performed experiments, analyzed data, and prepared the manuscript. KK, BCD, and VD analyzed data, performed quantitation, and revised the manuscript. SB conceptualized and supervised the project and wrote and revised the manuscript. VD, AS, and SB provided resources and revised the manuscript.

\section{Acknowledgments}

The authors would like to acknowledge Oliver Umland at the Diabetes Research Intstitute, University of Miami, for his support with flow cytometry. The authors would also like to thank Govindi Samaranayake and Clara Trocolli in Priyamvada Rai's lab for help with construction of the tet-inducible GFAT1 clone. This study was funded by NIH grants R01-CA170946 and R01-CA124723 (to AS), NIH grant R01-CA184274 (to SB), start-up support from the Sylvester Comprehensive Cancer Center, University of Miami (to SB), Katherine and Robert Goodale foundation support (to AS), and support from Minneamrita Therapeutics LLC (to AS).

Address correspondence to: Sulagna Banerjee, Department of Surgery, Biomedical Research Building, Rm 508, University of Miami, 1551 Northwest 10th Avenue, Florida 33136, USA. Phone: 305.243.8242; Email: sulagna.banerjee@med.miami.edu. 
1. Jemal A, Bray F, Center MM, Ferlay J, Ward E, Forman D. Global cancer statistics. CA Cancer J Clin. 2011;61(2):69-90.

2. [No authors listed]. Cancer Stat Facts: Pancreatic Cancer. National Cancer Institute. https:// seer.cancer.gov/statfacts/html/pancreas.html. Accessed November 21, 2019.

3. Chiorean EG, Coveler AL. Pancreatic cancer: optimizing treatment options, new, and emerging targeted therapies. Drug Des Devel Ther. 2015;9:3529-3545.

4. Neesse A, et al. Stromal biology and therapy in pancreatic cancer. Gut. 2011;60(6):861-868.

5. Kabacaoglu D, Ciecielski KJ, Ruess DA, Algül H. Immune checkpoint inhibition for pancreatic ductal adenocarcinoma: current limitations and future options. Front Immunol. 2018;9:1878.

6. Martinez-Bosch N, Vinaixa J, Navarro P. Immune evasion in pancreatic cancer: from mechanisms to therapy. Cancers (Basel). 2018;10(1):E6.

7. Elahi-Gedwillo KY, Carlson M, Zettervall J, Provenzano PP. Antifibrotic therapy disrupts stromal barriers and modulates the immune landscape in pancreatic ductal adenocarcinoma. Cancer Res. 2019;79(2):372-386.

8. Zhang Y, et al. Myeloid cells are required for PD-1/PD-L1 checkpoint activation and the establishment of an immunosuppressive environment in pancreatic cancer. Gut. 2017;66(1):124-136.

9. Jacobetz MA, et al. Hyaluronan impairs vascular function and drug delivery in a mouse model of pancreatic cancer. Gut. 2013;62(1):112-120.

10. Banerjee S, et al. Impaired synthesis of stromal components in response to minnelide improves vascular function, drug delivery, and survival in pancreatic cancer. Clin Cancer Res. 2016;22(2):415-425.

11. Provenzano PP, Cuevas C, Chang AE, Goel VK, Von Hoff DD, Hingorani SR. Enzymatic targeting of the stroma ablates physical barriers to treatment of pancreatic ductal adenocarcinoma. Cancer Cell. 2012;21(3):418-429.

12. Doherty GJ, Tempero M, Corrie PG. HALO-109301: a Phase III trial of PEGPH20 (with gemcitabine and nab-paclitaxel) in hyaluronic acidhigh stage IV pancreatic cancer. Future Oncol. 2018;14(1):13-22.

13. Rhim AD, et al. Stromal elements act to restrain, rather than support, pancreatic ductal adenocarcinoma. Cancer Cell. 2014;25(6):735-747.

14. Fukunaga A, et al. CD8+ tumor-infiltrating lymphocytes together with CD4+ tumor-infiltrating lymphocytes and dendritic cells improve the prognosis of patients with pancreatic adenocarcinoma. Pancreas. 2004;28(1):e26-e31.

15. Ino $Y$, et al. Immune cell infiltration as an indicator of the immune microenvironment of pancreatic cancer. Br J Cancer. 2013;108(4):914-923.

16. Stromnes IM, et al. Targeted depletion of an MDSC subset unmasks pancreatic ductal adenocarcinoma to adaptive immunity. Gut. 2014;63(11):1769-1781.

17. Zhang Y, et al. CD4+ T lymphocyte ablation prevents pancreatic carcinogenesis in mice. Cancer Immunol Res. 2014;2(5):423-435.

18. von Bernstorff W, et al. Systemic and local immunosuppression in pancreatic cancer patients. Clin Cancer Res. 2001;7(3 Suppl):925s-932s.

19. Franciszkiewicz K, Boissonnas A, Boutet M, Combadière C, Mami-Chouaib F. Role of chemokines and chemokine receptors in shaping the effector phase of the antitumor immune response. Cancer Res. 2012;72(24):6325-6332.

20. Friedl P, Weigelin B. Interstitial leukocyte migration and immune function. Nat Immunol. 2008;9(9):960-969.

21. Friedl P, Bröcker EB, Zänker KS. Integrins, cell matrix interactions and cell migration strategies: fundamental differences in leukocytes and tumor cells. Cell Adhes Commun. 1998;6(2-3):225-236.

22. Hartmann $\mathrm{N}$, et al. Prevailing role of contact guidance in intrastromal T-cell trapping in human pancreatic cancer. Clin Cancer Res. 2014;20(13):3422-3433.

23. Bohaumilitzky L, Huber AK, Stork EM, Wengert $\mathrm{S}$, Woelfl F, Boehm H. A trickster in disguise: hyaluronan's ambivalent roles in the matrix. Front Oncol. 2017;7:242.

24. McCarthy JB, El-Ashry D, Turley EA. Hyaluronan, cancer-associated fibroblasts and the tumor microenvironment in malignant progression. Front Cell Dev Biol. 2018;6:48.

25. Chanmee T, et al. Hyaluronan production regulates metabolic and cancer stem-like properties of breast cancer cells via hexosamine biosynthetic pathway-coupled HIF-1 signaling. J Biol Chem. 2016;291(46):24105-24120.

26. Vigetti D, Viola M, Karousou E, De Luca G, Passi A. Metabolic control of hyaluronan synthases. Matrix Biol. 2014;35:8-13.

27. Asthana A, Ramakrishnan P, Vicioso Y, Zhang K, Parameswaran R. Hexosamine biosynthetic pathway inhibition leads to AML cell differentiation and cell death. Mol Cancer Ther. 2018;17(10):2226-2237.

28. Zhang W, et al. GFPT2-expressing cancerassociated fibroblasts mediate metabolic reprogramming in human lung adenocarcinoma. Cancer Res. 2018;78(13):3445-3457.

29. Li L, et al. High expression of GFAT1 predicts unfavorable prognosis in patients with hepatocellular carcinoma. Oncotarget. 2017;8(12):19205-19217.

30. Vasseur S, Manié SN. ER stress and hexosamine pathway during tumourigenesis: A pas de deux? Semin Cancer Biol. 2015;33:34-39.

31. Ferrer CM, Reginato MJ. Sweet connections: O-GlcNAcylation links cancer cell metabolism and survival. Mol Cell Oncol. 2015;2(1):e961809.

32. Sharma NS, et al. O-GlcNAc modification of Sox 2 regulates self-renewal in pancreatic cancer by promoting its stability. Theranostics. 2019;9(12):3410-3424.

33. Banerjee S, et al. Triptolide-induced cell death in pancreatic cancer is mediated by O-GlcNAc modification of transcription factor Sp1. J Biol Chem. 2013;288(47):33927-33938.

34. Lyons SD, Sant ME, Christopherson RI. Cytotoxic mechanisms of glutamine antagonists in mouse L1210 leukemia. J Biol Chem. 1990;265(19):11377-11381.

35. Cervantes-Madrid D, Romero Y, DueñasGonzález A. Reviving lonidamine and 6-Diazo-5oxo-L-norleucine to be used in combination for metabolic cancer therapy. Biomed Res Int. 2015;2015:690492.

36. Hensley CT, Wasti AT, DeBerardinis RJ. Glutamine and cancer: cell biology, physiology, and clinical opportunities. JClin Invest. 2013;123(9):3678-3684.
37. Rais R, et al. Discovery of 6-Diazo-5-oxo-Lnorleucine (DON) prodrugs with enhanced CSF delivery in monkeys: a potential treatment for glioblastoma. JMed Chem. 2016;59(18):8621-8633.

38. Lemberg KM, Vornov JJ, Rais R, Slusher BS. We're not "DON" yet: optimal dosing and prodrug delivery of 6-Diazo-5-oxo-L-norleucine. Mol Cancer Ther. 2018;17(9):1824-1832.

39. Son J, et al. Glutamine supports pancreatic cancer growth through a KRAS-regulated metabolic pathway. Nature. 2013;496(7443):101-105.

40. Thiruvengadam SS, Chuang J, Huang R, Girotra M, Park WG. Chronic pancreatitis changes in high-risk individuals for pancreatic ductal adenocarcinoma. Gastrointest Endosc. 2019;89(4):842-851.e1.

41. Jiang M, et al. O-GlcNAcylation promotes colorectal cancer metastasis via the miR-101O-GlcNAc/EZH2 regulatory feedback circuit. Oncogene. 2019;38(3):301-316.

42. Ferrer CM, Lu TY, Bacigalupa ZA, Katsetos CD, Sinclair DA, Reginato MJ. O-GlcNAcylation regulates breast cancer metastasis via SIRT1 modulation of FOXM1 pathway. Oncogene. 2017;36(4):559-569.

43. Ali A, et al. O-GlcNAcylation of NF- $\kappa$ B promotes lung metastasis of cervical cancer cells via upregulation of CXCR4 expression. Mol Cells. 2017;40(7):476-484

44. Mielgo A, Schmid MC. Impact of tumour associated macrophages in pancreatic cancer. $B M B$ Rep. 2013;46(3):131-138.

45. Nakajima C, et al. A role of interferon-gamma (IFN-gamma) in tumor immunity: $\mathrm{T}$ cells with the capacity to reject tumor cells are generated but fail to migrate to tumor sites in IFN-gamma-deficient mice. Cancer Res. 2001;61(8):3399-3405.

46. Hu H, Hang JJ, Han T, Zhuo M, Jiao F, Wang LW. The M2 phenotype of tumor-associated macrophages in the stroma confers a poor prognosis in pancreatic cancer. Tumour Biol. 2016;37(7):8657-8664.

47. Peranzoni E, et al. Macrophages impede CD8 T cells from reaching tumor cells and limit the efficacy of anti-PD-1 treatment. Proc Natl Acad Sci US A. 2018;115(17):E4041-E4050.

48. Li T, Le A. Glutamine metabolism in cancer. $A d v$ Exp Med Biol. 2018;1063:13-32.

49. Cluntun AA, Lukey MJ, Cerione RA, Locasale JW. Glutamine metabolism in cancer: understanding the heterogeneity. Trends Cancer. 2017;3(3):169-180.

50. Szeliga M, Ćwikła J, Obara-Michlewska M, Cichocki A, Albrecht J. Glutaminases in slowly proliferating gastroenteropancreatic neuroendocrine neoplasms/tumors (GEP-NETs): Selective overexpression of mRNA coding for the KGA isoform. Exp Mol Pathol. 2016;100(1):74-78.

51. Yang C, et al. High expression of GFAT1 predicts poor prognosis in patients with pancreatic cancer. Sci Rep. 2016;6:39044.

52. Weinberg F, et al. Mitochondrial metabolism and ROS generation are essential for Krasmediated tumorigenicity. Proc Natl Acad Sci US A. 2010;107(19):8788-8793.

53. Gaglio D, et al. Oncogenic K-Ras decouples glucose and glutamine metabolism to support cancer cell growth. Mol Syst Biol. 2011;7:523.

54. Ying H, et al. Oncogenic Kras maintains pancreatic tumors through regulation of anabolic 
glucose metabolism. Cell. 2012;149(3):656-670. 55 . Sousa CM, Kimmelman AC. The complex landscape of pancreatic cancer metabolism. Carcinogenesis. 2014;35(7):1441-1450.

56. Zachara NE, Hart GW. O-GlcNAc a sensor of cellular state: the role of nucleocytoplasmic glycosylation in modulating cellular function in response to nutrition and stress. Biochim Biophys Acta. 2004;1673(1-2):13-28.

57. Zachara NE, Hart GW. O-GlcNAc modification: a nutritional sensor that modulates proteasome function. Trends Cell Biol. 2004;14(5):218-221.

58 . Ge J, et al. Glutaminolysis promotes collagen translation and stability via $\alpha$-ketoglutarate-mediated mTOR activation and proline hydroxylation. Am J Respir Cell Mol Biol. 2018;58(3):378-390.

59. Olive KP, et al. Inhibition of Hedgehog signaling enhances delivery of chemotherapy in a mouse model of pancreatic cancer. Science. 2009;324(5933):1457-1461.

60. Özdemir BC, et al. Depletion of carcinomaassociated fibroblasts and fibrosis induces immunosuppression and accelerates pancreas cancer with reduced survival. Cancer Cell. 2014;25(6):719-734.

61. Dauer P, et al. Inactivation of cancer-associated- fibroblasts disrupts oncogenic signaling in pancreatic cancer cells and promotes its regression. Cancer Res. 2018;78(5):1321-1333.

62. Nagathihalli NS, et al. Signal transducer and activator of transcription 3, mediated remodeling of the tumor microenvironment results in enhanced tumor drug delivery in a mouse model of pancreatic cancer. Gastroenterology. 2015;149(7):19321943.e9.

63. Yuzhalin AE, Lim SY, Kutikhin AG, GordonWeeks AN. Dynamic matrisome: ECM remodeling factors licensing cancer progression and metastasis. Biochim Biophys Acta Rev Cancer. 2018;1870(2):207-228.

64. Bertero T, et al. Tumor-stroma mechanics coordinate amino acid availability to sustain tumor growth and malignancy. Cell Metab. 2019;29(1):124-140.e10.

65. Tumeh PC, et al. PD-1 blockade induces responses by inhibiting adaptive immune resistance. Nature. 2014;515(7528):568-571.

66. Sklaroff RB, Casper ES, Magill GB, Young CW. Phase I study of 6-diazo-5-oxo-L-norleucine (DON). Cancer Treat Rep. 1980;64(12):1247-1251.

67. Michl P, Krug S. Overcoming immune evasion in pancreatic cancer: the combination matters. Gut.
2018;67(6):997-999.

68. Sangwan V, et al. Primary and liver metastasisderived cell lines from KrasG12D; Trp53R172H; Pdx-1 Cre animals undergo apoptosis in response to triptolide. Pancreas. 2015;44(4):583-589.

69. Sharon Y, Alon L, Glanz S, Servais C, Erez N. Isolation of normal and cancer-associated fibroblasts from fresh tissues by Fluorescence Activated Cell Sorting (FACS). J Vis Exp. 2013;(71):e4425.

70. Apte MV, et al. Periacinar stellate shaped cells in rat pancreas: identification, isolation, and culture. Gut. 1998;43(1):128-133.

71. Ao Z, et al. Identification of cancer-associated fibroblasts in circulating blood from patients with metastatic breast cancer. Cancer Res. 2015;75(22):4681-4687.

72. Banerjee S, et al. CD133+ tumor initiating cells in a syngenic murine model of pancreatic cancer respond to Minnelide. Clin Cancer Res. 2014;20(9):2388-2399.

73. Wiederschain D, et al. Single-vector inducible lentiviral RNAi system for oncology target validation. Cell Cycle. 2009;8(3):498-504.

74. Wee S, et al. PTEN-deficient cancers depend on PIK3CB. Proc Natl Acad Sci U S A. 2008;105(35):13057-13062. 